\title{
The social stigma of unemployment: consequences of stigma consciousness on job search attitudes, behaviour and success
}

\author{
Gerhard Krug ${ }^{1,2^{*}} \mathbb{D}$, Katrin Drasch ${ }^{2}$ and Monika Jungbauer-Gans ${ }^{3}$
}

\begin{abstract}
Studies show that the unemployed face serious disadvantages in the labour market and that the social stigma of unemployment is one explanation. In this paper, we focus on the unemployed's expectations of being stigmatized (stigma consciousness) and the consequences of such negative expectations on job search attitudes and behaviour. Using data from the panel study "Labour Market and Social Security" (PASS), we find that the unemployed with high stigma consciousness suffer from reduced well-being and health. Regarding job search, the stigmatized unemployed are more likely to expect that their chances of re-employment are low, but in contrast, they are more likely to place a high value on becoming re-employed. Instead of becoming discouraged and passive, we find that stigmatized unemployed individuals increase their job search effort compared to other unemployed individuals. However, despite their higher job search effort, the stigma-conscious unemployed do not have better re-employment chances.
\end{abstract}

Keywords: Unemployment, Social stigma, Stigma consciousness, Job search, Expectancy-value theory

JEL Classification: J64, J68, J78

\section{Introduction}

Unemployment is associated with adverse consequences. Empirical evidence has been presented for social exclusion (Hirseland and Ramos Lobato 2014), network withdrawal (Jones 1988), marital dissolution (Hansen 2005), financial shame (Rantakeisu et al. 1999), ill health (Krug and Eberl 2018), as well as reduced wage levels (Gangl 2004), reduced well-being (Mousteri et al. 2018), even after re-employment. For many of these consequences, social stigma is considered one of the central mechanisms (for an overview, see Brand 2015). The social stigma literature, in contrast, rarely addresses the stigma of unemployment, instead focussing on the stigma of mental or physical illness (Baumann 2007; Scambler 2009), race (Mosley and Rosenberg 2007; Pinel et al. 2005; Sigelman and Tuch 1997), ethnicity (Binggeli et al. 2014), sexual orientation (Herek 2010; Mattocks et al. 2015), etc. If at

\footnotetext{
*Correspondence: gerhard.krug@iab.de; gerhard.krug@fau.de

${ }^{1}$ Institute for Employment Research (IAB), Regensburger Str. 104,

90478 Nuremberg, Germany

Full list of author information is available at the end of the article
}

all, unemployment is only addressed as a potential consequence of other social stigmas such as mental illness or history of incarceration (cf., Link and Phelan 2001; LeBel 2008; Karren and Sherman 2012).

However, there is no doubt that in modern welfare states, there is a number of stereotypical beliefs regarding the attitudes of the unemployed to work and other personal shortcomings that are seen as the main reason for why individuals are getting and remain unemployed (Oschmiansky et al. 2003; McFadyen 1998). One strand of literature in labour market research explicitly addresses unemployment as a social stigma and shows that it might be these stereotypical beliefs that can hinder the unemployed from getting a job. This literature focusses on the discrimination of the unemployed, especially by firms during the hiring process. This research consistently shows that even if they had the same qualifications and competences as employed applicants, the unemployed and especially the long-term unemployed have significantly lower chances of getting hired. In a recent survey using German data, Rebien and Rothe (2018) showed that discrimination against the unemployed is 
very common. The authors found that only $14 \%$ of German firms would fill current vacancies with unemployed applicants irrespective of their unemployment duration. Thirty-four percent of these firms would accept such applications only if the applicants were unemployed for less than 1 year. The unemployment discrimination literature mostly focusses on firms' behaviour towards the unemployed. As a result, we have ample empirical evidence regarding the demand side of the matching process but not so much on the supply side. This empirical one-sidedness can leave the impression that the targets of unemployment stigma are only passive victims of potential employers' discriminatory hiring behaviour.

In this paper, we aim to contribute to the existing literature by illuminating the role of individuals' experience with social unemployment stigma in shaping their behavioural responses towards being stigmatized. Specifically, we ask whether this experience helps to co-create the adverse re-employment chances by influencing the job search behaviour and job search success, as is suggested by several authors. To do this, we apply the concept of stigma consciousness to the context of unemployment. This concept explicitly focusses on whether stigmatized individuals internalize the expectation of being stereotyped in social interactions. Stigma consciousness is defined as the extent to which individual targets of specific stereotypes "focus on their stereotyped status and believe it pervades their life experiences" (Pinel et al. 2005: 482). In other contexts of social stigma (e.g., gender, sexual orientation, or disabilities), it has been shown that the degree to which individuals perceive themselves to be subjected to stigmatization significantly influences their behaviour. For our analysis in the context of unemployment, we use data from the panel study "Labour Market and Social Security" (PASS) (Trappmann et al. 2013). A new scale was developed and implemented in 2013 to measure stigma consciousness among the unemployed (Gurr and Jungbauer-Gans 2013). We first corroborate that a higher stigma consciousness is associated with lower subjective well-being and lower health satisfaction. Based on expectancy-value theory, we find that those who are more stigma conscious have lower expectations of finding a job but highly value obtaining a job. However, our main result is that instead of leading to a reduced job search effort, those unemployed with a higher stigma consciousness are more likely to engage in an active job search, use more job search methods, spend more time searching for jobs, etc. Despite these positive associations with job search effort, we find that high stigma consciousness is not correlated with re-employment chances.

The remainder of this paper proceeds as follows. Section 2 discusses our definition of social stigma and stigma consciousness. Section 3 presents our theoretical considerations and how we derived our hypotheses. Section 4 presents a literature review regarding the role of unemployment stigma in labour market outcomes. Section 5 outlines our data, operationalization and analytical strategy. Section 6 presents the results of our study and discusses some limitations. Section 7 concludes with a summary and discussion of our results.

\section{Definitions of stigma and stigma consciousness}

The concept of stigma has received considerable interest in social science research, but as Link and Phelan (2001) remark, there is great variability in the definitions applied by different researchers. According to the seminal treatment of the topic by sociologist Erving Goffman (1963: 3 ), stigma is "an attribute that is deeply discrediting" and leads to negative and often hostile behaviour towards the stigmatized. Goffman distinguishes three types of stigmatizing conditions: tribal identities (e.g., ethnicity, religion, nationality or gender), abominations of the body (e.g., physical disabilities or deformities) and blemishes of character (e.g., mental illness, addiction, or previous incarceration). Unemployment can be regarded as an example of stigma of character, where the stigmatized are considered individuals with a "weak will, domineering or unnatural passions, treacherous and rigid beliefs, and dishonesty" (Goffman 1963: 4).

Several researchers have expanded upon Goffman's definition of stigma. For example, Link and Phelan (2001) propose conceptualizing stigma as the interrelation of four processes. First, the differences among members of society are distinguished and labelled. Second, these differences are associated with negative attributes. Third, the labels attached to differences imply a separation of "them" from "us". Fourth, the labelled person experiences a loss of status and discrimination. "Thus, we apply the term stigma when elements of labelling, stereotyping, separation, status loss and discrimination co-occur in a power situation that allows them to unfold" (Link and Phelan 2001: 367). This definition has been criticized by Deacon (2006) because it sees discrimination as an integral part of the stigma concept. In contrast, she defines stigma independent of discrimination. If we apply the definition to the context of unemployment, stigma can be defined as the following social process: Unemployment is construed as preventable and controllable; "immoral" behaviours causing unemployment are identified; these behaviours are associated with carriers of the characteristic in other groups, drawing on existing social constructs of the "other"; the unemployed are thus blamed for their situation; status loss is projected onto the "other", which may (or may not) result in disadvantage to them (adapted from Deacon 2006: 421). In contrast to Link and Phelan (2001, see also Besley and Coate 1992) 
Deacon (2006: 421) points out that being stigmatized is not automatically associated with disadvantages caused by discrimination. Furthermore, stigmatization can lead to disadvantages even in the absence of discrimination because it can have negative consequences for the selfconcept and actions of the stigmatized individuals.

The reason for disadvantages that are independent of discrimination is that the stigma is internalized by the stigmatized individuals and manifests itself in self-stigma ${ }^{1}$ (Bos et al. 2013; Pryor and Reeder 2011). According to Stuber and Schlesinger (2006), self-stigma combines two aspects, i.e., identity and treatment stigma. Identity stigma refers to the internalization of negative labels and stereotypes by the stigmatized individual, resulting in negative self-characterizations. Treatment stigma, in turn, refers to expectations about negative treatment by others. Both identity and treatment stigma are part of the self-stigma and therefore to be distinguished from actual discrimination because both are based on the perceptions of the stigmatized themselves. "For example, administrative practices that are not inherently discriminatory (such as questions about personal finances or living arrangements) may be interpreted by potential recipients as such" (Stuber and Schlesinger 2006: 935).

In the empirical analysis below, we apply the concept of stigma consciousness to the context of unemployment. Several authors (e.g., Taylor et al. 1994, cited after LeBel 2008) have shown that there is a discrepancy between group discrimination and the extent to which individuals personally experience discrimination. While some stigmatized individuals do, others do not attribute negative outcomes to stereotypes and discriminations. To cover these differences, Pinel (1999) developed and validated a 10-item 'Stigma Consciousness Questionnaire' (SCQ) for several stereotyped groups (e.g., women, lesbians, gay men, African Americans, and Latinos/Latinas). Stigma consciousness reflects the extent to which individual targets of specific stereotypes "focus on their stereotyped status and believe it pervades their life experiences" (Pinel et al. 2005: 482). According to Pinel (1999), consciousness of the stigma is a key determinant of the stigmatized individual's behavioural reactions. Stigma consciousness is argued to increase the perception of being discriminated against and to heighten the belief that group membership influences social interactions and experience (Guyll et al. 2010). Negative feedback from

\footnotetext{
${ }^{1}$ Other manifestations are public stigma (shared attitudes and behaviour of a society towards the stigmatized), structural stigma (legitimization of a social stigma by being embedded in a society's institutions) and stigma by association (people's reaction of being associated with a stigmatized person) (Pryor and Reeder 2011).
}

others is more often interpreted as discriminatory. Thus, stigma consciousness is viewed as a mechanism mediating the association between group membership and negative outcomes.

\section{Consequences of stigma of unemployment: theoretical considerations and hypotheses}

Several scholars have examined the consequences of internalizing stigmatizing stereotypes, but the bulk of research focusses on contexts such as ethnicity, gender, medical conditions, sexual orientation or history of incarceration (see e.g., LeBel 2008). In this paper, we are interested in the stigma of unemployment and its consequences. Changes in subjective well-being and health are among the most obvious consequences of any type of stigma (Hatzenbuehler et al. 2013; Markowitz 1998; Rosenfield 1997), and the stigma of unemployment should be no exception. However, empirical evidence is scarce, but O'Donnell et al. (2015) found that anticipated stigma, which is a measure similar to stigma consciousness, has a negative impact on psychological distress and physical health.

We follow this strand of research and argue that stigmatization consciousness is directly connected to subjective well-being and health. Therefore, our first two hypotheses are concerned with the proposition that the stigmatized suffer from their status as unemployed more than the non-stigmatized as follows:

H1 The higher the stigma consciousness among the unemployed, the lower their subjective well-being.

H2 The higher the stigma consciousness among the unemployed, the lower their subjective health.

However, the main concern of the present analysis is the role of stigma consciousness for job search. According to Goffman (1963), an important dimension of the stigma influencing how it is perceived by the respective targets, is its visibility. As an example of a stigma of character (e.g., Gurr and Jungbauer-Gans 2017), unemployment is a stigma that is not highly visible and therefore often concealable. The unemployed are therefore "discreditable" instead of "discredited". Thus, in social situations, the unemployed can often choose whether they disclose information regarding their status. This is not the case during the job search because to obtain reemployment, per definition, the unemployed have to disclose their status to other individuals.

Job search activities such as visiting the unemployment agency, asking friends for job leads, and attending job interviews make it difficult to conceal one's status as unemployed and are experienced as humiliating and 
potentially lead to rejection (Letkemann 2002). Therefore, reducing job search effort could be a viable strategy to avoid being stigmatized. The literature on welfare stigma (cf., Andrade 2002) even assumes that some of the unemployed will forego welfare benefits they are entitled to in order to avoid their stigma being made visible (Yaniv 1997; Moffitt 1983; Loewenberg 1981). According to Sherman (2013), for those who do not have other financial options or find themselves unable to get a job despite their best efforts, the eventual acceptance of welfare benefits often leads to self-hatred, shame, and depression. Kerbo (1976) observes that welfare benefit claimants exhibit lower job search activity than nonclaimants and attribute this behaviour to discouragement. He argues that those who felt highly stigmatized because they received welfare were also most likely to be the most passive. Heslin et al. (2012) develop a theoretical model that relates the labour market experience of members of ethnic minorities to the becoming discouraged workers, i.e., wanting to work but not looking for employment due to negative experiences. They argue that due to the stigma attached to the minority status (lazy, untrustworthy, etc.), they fare worse in the recruitment and selection process of employers. This experience will make them prone to become discouraged workers because among others, it leads to learned helplessness (Seligman 1975), that is, they become passive and no longer try to improve the negative situation that they perceive as uncontrollable. According to Abramson et al. (1978), individuals are more disposed to react with learned helplessness if they see the reason for their negative experience in themselves.

According to Pinel (1999), an important predictor of whether individuals avoid situations in which stigma is salient is stigma consciousness. She found that those with higher stigma consciousness are more likely to avoid situations where they expect to be stereotyped. For example, compared to those with low stigma consciousness, female workers with high stigma consciousness were more likely to intend to and actually leave their job (Pinel and Paulin 2005). Stigma consciousness can cause greater experience of stereotype threat, raise the level of perceived prejudices and feelings of rejection, and reduce the person's sense of control and self-esteem (Wang et al. 2012). Wang and her colleagues show that a person with high stigma consciousness more often views subtle bias as discrimination and becomes angrier.

Thus, there seems to be a consensus in the literature that stigmatization and specifically stigma consciousness should result in reduced job search efforts. However, there are also some indications in the literature that this view might be too one-sided. Based on stress theory, Miller and Kaiser (2001) note that individuals tend to have two options that the authors call engagement and disengagement behaviour. While the above discussion highlights the potential of social stigma of unemployment to result in disengagement or avoidance behaviour, in other contexts of stigmatization (e.g., mental health), it has been shown that some stigmatized individuals choose engagement behaviour, e.g., raising awareness of social stigma or in the form of problem solving. For example, the above cited Wang et al. (2012) also find that the high stigma conscious are more often willing to engage in collective action. By directly referencing to the stigma of unemployment, Bretschneider (2014) draws a similar conclusion using group identity theory (Tajfel and Turner 1979). She argues that to the degree that group boundaries are perceived as permeable and social mobility between groups is possible, individuals are more likely to attempt to obtain a more positive sense of self by changing groups. An important way for the unemployed to achieve this goal is by proactively engaging in job search.

To develop testable predictions regarding the potentially ambiguous effect of stigma consciousness on the job search effort, we can draw upon the expectancy-value theory (Vroom 1964). This theory was first applied to the job search process by Feather (1982), and here we extend this theory to incorporate stigma consciousness among the unemployed. Expectancy-value theory assumes that the level of job search effort is determined by two factors, i.e., expectations and value. The first determinant of job search effort expectation refers to the expectations that specific behaviour, such as the job search in our case, will result in the desired outcome, such as obtaining gainful employment. Expectancy-value theory predicts that individuals with high expectation that their job search will be successful will exert more effort in the job search. Regarding the social stigma of unemployment, we hypothesize that their expectations of meeting negative stereotypes during the job search lead the unemployed with higher ratings on the stigma consciousness scale to have lower expectations of succeeding in the job search.

H3 The higher the stigma consciousness among the unemployed, the lower their expectations of their successful re-employment chances.

Value is the second determinant of effort and refers to the degree to which the desired outcome of the job search, i.e., becoming re-employed, is valued by the unemployed. The central behavioural assumption is that the higher the subjective value of the desired outcome, the higher the effort exerted to obtain this outcome. We assume that unemployed individuals with high levels of stigma consciousness are more likely to place a high value 
on re-employment possibly because the stigmatized are more likely to suffer from adverse consequences due to their status as unemployed compared to other unemployed persons. Our next hypothesis is as follows:

H4 The higher the stigma consciousness among the unemployed, the more value they place on employment.

Given that expectancy-value theory assumes that both expectations and value determine the job search effort, we consider predictions regarding the effect of stigma consciousness on job search effort ambiguous. Regarding expectations, high stigma consciousness should result in lower job search effort; however, regarding the value of re-employment, a higher job search effort is expected. Thus, the overall effect depends on the relative importance of either of the two factors, resulting in two competing hypotheses.

H5a The higher the stigma consciousness among the unemployed, the lower the job search effort.

$H 5 b$ The higher the stigma consciousness among the unemployed, the higher the job search effort.

Several scholars assume that the actual re-employment chances are crucially determined by the intensity of the job search effort as follows: the higher the job search effort, the higher the probability of obtaining adequate and acceptable job offers (see e.g., Mortensen 1986). Therefore, for job search success, we also posit two opposing hypotheses. We expect that stigma consciousness has negative effects on job search success in the case of reduced effort, but in contrast, we expect positive effects in the case of increased job search effort.

H6a The higher the stigma consciousness among the unemployed, the lower is the job search success.

$H 6 b$ The higher the stigma consciousness among the unemployed, the higher is the job search success.

\section{Literature review}

Empirical evidence regarding the effect of unemployment stigma on job search behaviour is scarce. In contrast, there is ample and convincing evidence regarding discrimination of the unemployed in employers' hiring behaviour and so-called true unemployment state dependence. In the following, we review the part of the literature that explicitly connects their results to unemployment stigma as the key explanatory mechanism.
Several studies find that firms are reluctant to fill vacancies with an unemployed job seeker and explain this finding by the prevalence of unemployment stigma. In a correspondence experiment, Oberholzer-Gee (2008) observes that the callback rates for short-term unemployed individuals are even higher than those for employed job seekers, but if applicants are long-term unemployed, the callback rates decline. He finds that even after controlling for further characteristics of the supply side of a job offer, the duration of unemployment has a crucial negative effect on the likelihood of being invited to a job interview. Several studies have replicated and extended his results. Nüß (2017) finds that callbacks decline after 10 months of unemployment. Eriksson and Rooth (2014) do not find that past unemployment spells will lead to differential treatment regarding callbacks, nor will current short-term unemployment for up to 9 months. However, after this point, stigmatization effects arise, and callback rates decline. The authors also observe stronger stigma effects for men than for women. Ghayad (2014) reports that after more than 6 months of unemployment, work experience will no longer matter. The generally positive effect of an unemployed applicant's industry-specific human capital disappears, and callback rates are similar to those for unemployed persons without industry-specific human capital. Kroft et al. (2013) find that discrimination against the unemployed is common if labour markets are tight, and callback rates already start to decline after 6 months of unemployment. However, some studies do not observe any stigma effects. Nunley et al. (2017) find no effects of unemployment on callback, irrespective of labour market tightness. Similarly, Farber et al. (2015) do not find that unemployment reduces callback, but they do find reduced callback rates for applicants over the age of 50 . In a recent experiment, van Belle et al. (2017) also conclude that unemployment duration serves as a sorting criterion because employers view it as a signal of low motivation.

In a related stream of research, stigma is considered the reason for the so-called true state dependence in the duration of unemployment, i.e., the effect of past unemployment on one's current labour market status (e.g., Arulampalam 2001, 2002; Arulampalam et al. 2001; Heckman and Borjas 1980). Spurious state dependence means that unobserved differences between the unemployed create the impression that re-employment chances diminish with longer unemployment durations. By contrast, true state dependence means that the longer an individual is unemployed, the lower the chances of finding re-employment (e.g., van den Berg and van Ours 1996). One explanation provided by the literature for how true state dependence arises is the stigmatization of the (long-term) unemployed by employers because they 
consider unemployment as a signal of low motivation or productivity (Vishwanath 1989).

For example, Biewen and Steffes (2010) find significant effects of past unemployment on the present unemployment risk; these effects decrease when unemployment rates are high. The authors consider this to be evidence of stigma effects because individual unemployment is less likely to be interpreted as a negative signal if unemployment is high and vice versa (see also Omori 1997). Ayllón (2013) reports similar results but also finds that if unemployment rates are high, discouragement effects counterbalance the lower stigma effect to some extent.

A third strand of literature is addressing the unemployed social experience as stigmatized but is more strongly focussed on psychological coping mechanisms or communication strategies of the unemployed as a reaction to their status as stigmatized. Knabe et al. (2018) analyse whether social networks can be a substitute for stigmatized unemployed to feel respected and appreciated. Gurr and Jungbauer-Gans (2017) focus on whether or not the unemployed have internalized society's view that the unemployed themselves are to be blamed for their situation. Similar, but with a stronger focus on job search requirements, Hirseland and Ramos Lobato (2014) found that the unemployed react to stigmatizing media discourse ("lazy unemployed") by either taking over the public opinion, by seeing themselves as an exception to the rule or by complying with the public demands for intensified job search effort. Research using the same data as that used in the following analysis yielded the following results: Lang and Gross (2017) find that unemployment stigma consciousness is determined by the strength of deviation, the scope of the norm's application and the intensity of formal social control; Gurr et al. (2018) find no effects of unemployment benefit sanctions on stigma consciousness; and Linden et al. (2018) find that being exempted from job search requirements due to ill health does not reduce stigma consciousness among the unemployed.

Overall, empirical evidence regarding how the social stigma of unemployment is related to job search attitudes, behaviour and re-employment success is scarce. To the best of our knowledge, the only studies intersecting with ours are a qualitative data analysis performed by Hirseland and Ramos Lobato (2014) and a quantitative paper published by Kerbo (1976); however, in both studies, no formal tests of these relationships were conducted.

\section{Data and method}

\subsection{Data and operationalizations}

In the following analysis, we use the German household panel study PASS (Trappmann et al. 2013), which began in 2007, and at the time of the writing of this manuscript, ten waves were available. PASS consists of two almost equally large subsamples, a probability sample drawn from all long-term unemployed persons registered with the German federal employment services and a random population sample. We use both samples for our analysis, but because our focus is on the unemployed, the registered unemployed sample dominates our analytical sample. Both subsamples of the PASS were refreshed several times and survey-provided weights are used in the analysis below to account for panel attrition. PASS collected data regarding (un-)employment histories retrospectively, and for each wave, detailed information about the current employment or unemployment situation is available.

Our operationalization of unemployment stigma relies on a scale that measures stigma consciousness among the unemployed who were part of wave 7 of PASS (Gurr and Jungbauer-Gans 2013). ${ }^{2}$ This scale builds upon Goffman's stigma concept and adapts a rather general psychological concept of gender stigmatization for the case of the unemployed (Pinel 1999). However, it also uses insights from other concepts and definitions described in the theoretical section of this article (e.g., Link and Phelan 2001). We exclude the item "I am trying to find a job as quickly as possible" because this item measures a concept similar to one of our dependent variables job search effort. We construct an index by aggregating all but one of the above mentioned stigma item. We normalize the values of the scale to range from 0 (no stigmatization) to 10 (maximum stigmatization). Table 3 in the appendix provides an overview of the items used in the scale. With a value of Cronbach's alpha of 0.73 , this scale exhibits acceptable reliability. The average value of the scale amounted to 5.09 with a standard deviation of 1.80 , indicating on average medium stigmatization and considerable variation between individuals.

To test Hypotheses 1 and 2, we use the PASS questions regarding life and health satisfaction, both of which are measured on an 11-point scale ("In general, how satisfied are you currently with your life overall?"; "How satisfied are you today with your health?”). To test Hypothesis 3,

\footnotetext{
${ }^{2}$ The scale was originally designed to derive different distinct factors of stigmatization and distinguish between factors pertaining expectations with respect to other unemployed persons (in-group) and the general population (out-group) and strategies of action (Gurr and Jungbauer-Gans 2013). However, neither the pretest of the study nor the main study of PASS wave 7 confirms these theoretical expectations, though other non-congruent factors (social relations, avoidance of situations, pressure to act, awareness of prejudices) develop. In the PASS dataset factor analyses only confirms these factors to some extent. This could either be due to the low number of cases in the pretest $(\mathrm{N}=104)$ or the fact that distinct factors are difficult to measure within the stigmatization framework. Therefore, and in line with Gurr et al. (2018) and Linden et al. (2018) we use a sum score for our analyses.
} 
we use a self-assessment of the unemployed's employment chances ("What do you think are your chances to find a new job in the next 6 months? good/quite good/ quite bad/bad"), which was presented to all unemployed individuals regardless of whether they searched for a job. $^{3}$ To test Hypothesis 4, we use a factor score obtained from a set of four items measured on a four-point scale concerning non-monetary and monetary motivations to work as the dependent variable. We maintained the three items $^{4}$ reflecting non-monetary motivation because these items loaded on a common factor, whereas the fourth item constituted an independent factor.

In Hypothesis $5 \mathrm{a} / \mathrm{b}$, job search effort is the dependent variable. We use several different measures available in PASS to cover a wide range of potential indicators of higher or lower job search effort (see Table 4 in Appendix for a detailed overview). Our first and most basic indicator uses binary information regarding whether the respondent actively searched for a job within the prior 4 weeks. A second indicator is the sum of all job search methods actively used in the last 4 weeks. The third indicator uses additional information on the intensity with which a specific job search method was used. We use a sum score of all job search intensities from all job search methods used by the respondent. If a method is not used, intensity is coded as 0 . Indicator number four is the number of hours spent searching for a job. Indicator five measures the number of times during the last 4 weeks a respondent used one of the following ways to apply for a job: replied to job advertisements, placed an "employment wanted" advertisement with the newspaper, asked for a job at the company itself or submitted an application even though no job opening had been advertised. We set all indicators of the job search effort to zero for those respondents who left the labour force (e.g., "homemaker") because per definition, these individuals are not searching for a job, and not including them would systematically excludes the most discouraged unemployed.

In Hypotheses $6 \mathrm{a} / \mathrm{b}$, we are interested in the job search success. This is measured first as the number of job interviews a respondent had during the last 4 weeks. Second, we construct a dummy variable that assumes the value of one for those who hold a job and zero for those who are

\footnotetext{
${ }^{3}$ For several other items regarding job search expectations and attitudes, this was not the case.

${ }^{4}$ Let us now deal with the topic of work and gainful employment. Regardless of whether you currently work or not: To what extent do you agree to the following opinions on work? Please think very generally about working in a job. Please tell me whether you "strongly agree", "somewhat agree", "somewhat disagree" or "strongly disagree" with these opinions. "Having work is the most important thing in life."; "Work is important because it gives you the feeling of being a part of something/belonging."; "I would also like to work if I didn't need the money". (Official translation provided by PASS).
}

unemployed, have withdrawn from the labour force or are in any other state (e.g., retirement).

\subsection{Sample selection and analytical design}

Because stigma consciousness is measured only in one single wave, our analytical sample is in principle a crosssection. However, the stigma consciousness scale is embedded in an ongoing panel study. Therefore, while stigma consciousness is measured in wave 7 , the outcome variables are obtained from wave 8 of the PASS study. We include several important covariates (often time-constant or measured at wave 7) as control variables to account for socio-demographic information, including age, age squared, gender, marital status, migration background, educational attainment, place of residence (East/West Germany), household size, and household income, and a dummy variable representing the general population and the welfare benefit sample. The descriptive statistics (mean, standard deviation, minimum and maximum, and number of cases) of the dependent and independent variables are shown in Table 5 in Appendix.

In addition, we include information regarding previous employment status (unemployed, employed, or out of the labour force), which was measured in wave 6. Furthermore, we included unemployment duration and the number of previous unemployment spells, which were measured at wave 7 , as controls because these variables might influence the level of stigma consciousness. However, because we have no information regarding the level of stigma consciousness at the beginning of the unemployment episode, the current unemployment duration can also be an outcome of stigma consciousness, especially if reduced job search effort is the dominant reaction of the stigmatized. Alternatively, we can also consider unemployment duration and previous unemployment as alternative measures of unemployment stigma, albeit potentially confounded by the depreciation of human capital. Because of these alternative views, we also test our hypotheses without including the unemployment duration variables in the model. We find that the choice of including unemployment duration did not substantially influence our results (see Tables 6 and 7 in Appendix).$^{5}$

In summary, our analytical sample is selected as follows. From the 14,449 respondents in wave 7 , we restrict ourselves to 2448 respondents who were eligible to answer the stigma consciousness scale items. The main criterion for eligibility is registered unemployment

\footnotetext{
${ }^{5}$ Because unemployment duration is often seen to be negatively related to job-search effort, this stability might seem surprising. However, Schels and Bethmann (2018) found that for most unemployed, job search effort remains stable over time.
} 
during wave 7. We exclude individuals with missing stigma scale values, reducing the sample to 2286 individuals. Finally, we exclude those who dropped out in wave 8 (we used the PASS provided weights to account for this panel attrition), resulting in 1779 cases remaining. For Hypothesis 1, we arrive at our analytical sample of 1278 , which included individuals who were still unemployed or moved to the silent reserve ("homemaker") at the time of wave 8. For Hypothesis 2, the same sample is used if the number of job interviews is the dependent variable. If job-finding is the dependent variable, the sample size increases to 1573 because those who found employment between waves 7 and 8 will be included also. To avoid any further loss of cases and, thus, precision in our estimate, we multiply impute (Rubin 1987) the missing data in any of two cases. First, we impute the data if the data were missing due to item non-response. Second, we impute the data if the data were missing for respondents who entered the panel survey only during wave 7 because in this case, no information on several variables from wave 6 was available.

With our estimation strategy we follow standard procedures using liner regression, except for that we also use linear regression analyses for binary and ordinal outcome variables because the estimation of marginal effects after multiple imputations is cumbersome (see STATA multiple imputation reference manual release 13: 77). Thus, linear regressions are conducted to analyse life and health satisfaction (ordinal), re-employment expectation (ordinal), non-monetary employment motivation (continuous), active job search (binary), re-employment (binary) and job search intensity (continuous). All other outcomes are count data variables, i.e., hours spent searching, the number of job search methods, the number of applications and the number of job interviews, and we use negative binomial count data regressions, a method similar to Poisson regression, but more generally applicable (Cameron and Trivedi 2010).

\section{Results}

\subsection{Hypotheses tests}

This section presents the results of our empirical analysis. Please note that in the following, the terms "effect" and "coefficient" are used interchangeably and without intending to imply causality. See Sect. 7 for a discussion regarding what speaks for or against a causal interpretation of our regression results. In Table 1, Models 1-4 present the results of Hypotheses 1-4. Hypotheses 1 and 2 posit that people suffer from unemployment stigma, and therefore, higher stigma consciousness is associated with lower subjective well-being and lower health. As shown by Model 1 in Table 1, the coefficient of stigma consciousness is negative and statistically significant. The coefficient is -0.319 , indicating that a one point (or equivalently a $10 \%$ ) increase in stigma consciousness is associated with ca. 0.32-point reduction on the 11-point life-satisfaction scale. This reduction is only a slight one, because it accounts for a small part of the standard deviation of the dependent variable (see Table 5 in Appendix). The results of health satisfaction (Model 2 in Table 1) are similar, although the coefficient (0.196) is smaller. As shown, the respective coefficient is also statistically significant and negative, indicating that high stigma consciousness is associated with lower health satisfaction. Therefore, the data support hypotheses 1 and 2 .

Hypothesis 3 focusses on the unemployed's job expectations and posits that a negative association exists between stigma consciousness and self-perceived re-employment chances. Based on Model 3 in Table 1, the coefficient of the stigma consciousness scale on the 4-point scale of self-assessed chances of re-employment is -0.047 , which as predicted, is negative and statistically significant. The higher the unemployed's stigma consciousness, the lower their expectations of transitioning from unemployment to employment. Hypothesis 4 concerns the positive relationship between unemployment stigma and the value placed on re-employment. The respective coefficient (Model 4 in Table 1 ) is 0.125 , which is statistically significant. Thus, Hypotheses 3 and 4 are also supported by our data.

In Table 2, the results of Hypotheses $5 \mathrm{a} / \mathrm{b}$ and $6 \mathrm{a} / \mathrm{b}$ are presented. In both hypotheses, the theoretical prediction is ambiguous, allowing for both positive and negative associations between unemployment stigma and job search effort and job search success.

In Model 1 in Table 2, the dependent variable is active job search. Instead of the negative regression coefficient expected from Hypothesis 3a, Model 1 reports that stigma consciousness has a positive effect on whether unemployed individuals actively search for a job, and this effect is significant at the $0.1 \%$ level. For every additional point on the ten-point stigma consciousness scale, the probability of actively searching for a job increases by $2.6 \%$ points. Thus, two hypothetical individuals who are located at the opposite ends of the stigma consciousness scale could differ by $26 \%$ points in their probability of engaging in active job search, whereas the average probability of an active job search is approximately $52 \%$ in our analytical sample (see Table 5 in Appendix).

Model 2 in Table 2 extends the binary outcome variable by not only looking at active search but at the number of job search methods used during job search. With each additional point on the stigma consciousness scale, the number of methods significantly increases on average by 0.067 , which is also only a slight increase. Model 3 then takes into account that job search intensity can vary within each method used for job search and uses the sum score of all the respondents' values for each method used 
Table 1 Effects of unemployment stigma on subjective well-being (H1), health (H2), re-employment expectations (H3) and value placed on employment (H4)

\begin{tabular}{|c|c|c|c|c|}
\hline & $\begin{array}{l}\text { H1: Subjective well-being } \\
\text { Model } 1^{\text {a }}\end{array}$ & $\begin{array}{l}\text { H2: Health } \\
\text { Model } 2^{\mathrm{a}}\end{array}$ & $\begin{array}{l}\text { H3: Expectations } \\
\text { Model } 3^{a}\end{array}$ & $\begin{array}{l}\text { H4: Value } \\
\text { Model } 4^{\mathrm{a}}\end{array}$ \\
\hline Stigma consciousness (0-10) & $\begin{array}{l}-0.319^{* * *} \\
(0.033)\end{array}$ & $\begin{array}{l}-0.196^{\text {*** }} \\
(0.040)\end{array}$ & $\begin{array}{l}-0.047^{* * *} \\
(0.013)\end{array}$ & $\begin{array}{l}0.125^{* * *} \\
(0.017)\end{array}$ \\
\hline Sample (R: population sample) & $\begin{array}{l}-0.159 \\
(0.235)\end{array}$ & $\begin{array}{l}0.025 \\
(0.301)\end{array}$ & $\begin{array}{l}0.053 \\
(0.099)\end{array}$ & $\begin{array}{l}0.028 \\
(0.141)\end{array}$ \\
\hline \multicolumn{5}{|l|}{ Employment status at wave 6 (R: employed) } \\
\hline Unemployed & $\begin{array}{l}-0.328^{\#} \\
(0.186)\end{array}$ & $\begin{array}{l}-0.101 \\
(0.249)\end{array}$ & $\begin{array}{l}-0.196^{*} \\
(0.083)\end{array}$ & $\begin{array}{l}-0.215^{*} \\
(0.096)\end{array}$ \\
\hline Out of the labour force & $\begin{array}{l}-0.219 \\
(0.567)\end{array}$ & $\begin{array}{l}-0.287 \\
(0.750)\end{array}$ & $\begin{array}{l}-0.004 \\
(0.319)\end{array}$ & $\begin{array}{l}0.189 \\
(0.376)\end{array}$ \\
\hline Male & $\begin{array}{l}-0.347^{* *} \\
(0.115)\end{array}$ & $\begin{array}{l}-0.058 \\
(0.144)\end{array}$ & $\begin{array}{l}0.058 \\
(0.049)\end{array}$ & $\begin{array}{l}-0.172^{* *} \\
(0.060)\end{array}$ \\
\hline Unemployment duration (years) & $\begin{array}{l}0.003 \\
(0.014)\end{array}$ & $\begin{array}{l}-0.038^{*} \\
(0.019)\end{array}$ & $\begin{array}{l}-0.019^{* * *} \\
(0.005)\end{array}$ & $\begin{array}{l}0.013^{\#} \\
(0.007)\end{array}$ \\
\hline Number of previous unemployment episodes & $\begin{array}{l}-0.062 \\
(0.072)\end{array}$ & $\begin{array}{l}0.157^{\#} \\
(0.093)\end{array}$ & $\begin{array}{l}0.049 \\
(0.032)\end{array}$ & $\begin{array}{l}0.032 \\
(0.033)\end{array}$ \\
\hline Age & $\begin{array}{l}-0.098^{*} \\
(0.038)\end{array}$ & $\begin{array}{l}-0.288^{* * *} \\
(0.048)\end{array}$ & $\begin{array}{l}0.008 \\
(0.017)\end{array}$ & $\begin{array}{l}0.033 \\
(0.021)\end{array}$ \\
\hline $\mathrm{Age}^{2}$ & $\begin{array}{l}0.001^{*} \\
(0.000)\end{array}$ & $\begin{array}{l}0.003^{* * *} \\
(0.001)\end{array}$ & $\begin{array}{l}-0.000^{*} \\
(0.000)\end{array}$ & $\begin{array}{l}-0.000 \\
(0.000)\end{array}$ \\
\hline Migration background & $\begin{array}{l}0.343^{*} \\
(0.136)\end{array}$ & $\begin{array}{l}0.419^{*} \\
(0.176)\end{array}$ & $\begin{array}{l}0.088 \\
(0.063)\end{array}$ & $\begin{array}{l}0.019 \\
(0.074)\end{array}$ \\
\hline \multicolumn{5}{|l|}{ Education (R: elementary) (CASMIN) } \\
\hline Secondary & $\begin{array}{l}-0.062 \\
(0.131)\end{array}$ & $\begin{array}{l}-0.154 \\
(0.168)\end{array}$ & $\begin{array}{l}-0.006 \\
(0.059)\end{array}$ & $\begin{array}{l}-0.122^{\#} \\
(0.073)\end{array}$ \\
\hline Maturity & $\begin{array}{l}-0.103 \\
(0.176)\end{array}$ & $\begin{array}{l}0.476^{*} \\
(0.226)\end{array}$ & $\begin{array}{l}0.081 \\
(0.087)\end{array}$ & $\begin{array}{l}-0.351^{* * *} \\
(0.091)\end{array}$ \\
\hline Tertiary & $\begin{array}{l}-0.138 \\
(0.207)\end{array}$ & $\begin{array}{l}0.520^{*} \\
(0.241)\end{array}$ & $\begin{array}{l}0.125 \\
(0.088)\end{array}$ & $\begin{array}{l}-0.310^{* *} \\
(0.100)\end{array}$ \\
\hline Married & $\begin{array}{l}0.104 \\
(0.151)\end{array}$ & $\begin{array}{l}-0.161 \\
(0.187)\end{array}$ & $\begin{array}{l}-0.048 \\
(0.068)\end{array}$ & $\begin{array}{l}0.183^{*} \\
(0.085)\end{array}$ \\
\hline Household size & $\begin{array}{l}0.059 \\
(0.057)\end{array}$ & $\begin{array}{l}0.084 \\
(0.082)\end{array}$ & $\begin{array}{l}-0.034 \\
(0.030)\end{array}$ & $\begin{array}{l}0.000 \\
(0.037)\end{array}$ \\
\hline Household income (in $100 €$ ) & $\begin{array}{l}0.010 \\
(0.011)\end{array}$ & $\begin{array}{l}0.019 \\
(0.015)\end{array}$ & $\begin{array}{l}0.001 \\
(0.005)\end{array}$ & $\begin{array}{l}0.009 \\
(0.007)\end{array}$ \\
\hline West Germany & $\begin{array}{l}0.003 \\
(0.123)\end{array}$ & $\begin{array}{l}-0.462^{* *} \\
(0.153)\end{array}$ & $\begin{array}{l}0.075 \\
(0.055)\end{array}$ & $\begin{array}{l}-0.059 \\
(0.066)\end{array}$ \\
\hline Constant & $\begin{array}{l}10.008^{* * *} \\
(0.822)\end{array}$ & $\begin{array}{l}12.842^{* * *} \\
(1.090)\end{array}$ & $\begin{array}{l}2.688^{* * *} \\
(0.372)\end{array}$ & $\begin{array}{l}-1.492^{* *} \\
(0.471)\end{array}$ \\
\hline Observations & 1278 & 1278 & 1278 & 1278 \\
\hline
\end{tabular}

Weighted to account for panel attrition from waves 7 to 8 ; multiple imputations were performed by chained equations to account for missing data values (40 imputations, burn in period of 30 iterations). Goodness-of-fit tests not available after multiple imputations in STATA (see STATA multiple imputation reference manual release 13, p. 79)

Outcome variables in Models 1 to 4: life satisfaction (11-pt. scale); health satisfaction (11-pt. scale); self-assessed re-employment chances (4-pt. scale), and factor score of non-monetary motivation to work (continuous)

Robust standard errors are shown in parentheses; ${ }^{* *} p<0.001,{ }^{* *} p<0.01,{ }^{*} p<0.05,{ }^{*} p<0.10$

Estimation methods: ${ }^{\text {alinear regression }}$

as the dependent variable. Again, we observe a significantly positive but rather small effect. Our next indicator of job search effort is the number of hours spent searching for a job, where the coefficient of stigma consciousness is significant only at the $5 \%$ level. On average, an additional point on the stigma scale results in approximately 4.6 min per week
(60 $\mathrm{min}^{*} 0.077$ ) more time spent searching for a job. For Model 5, the number of times the unemployed applied for a job is the dependent variable. We observe a positive and statistically significant coefficient of 0.069 , indicating that a one-point increase in the stigma variable is associated with ca. 0.07 more applications. 
Table 2 Effects of unemployment stigma on job search effort (H5) and success (H6)

\begin{tabular}{|c|c|c|c|c|c|c|c|}
\hline & \multicolumn{5}{|c|}{ H5a/b: Job search effort } & \multicolumn{2}{|c|}{ H6a/b: Job search success } \\
\hline & $\begin{array}{l}\text { Active job search } \\
\text { (yes) }\end{array}$ & $\begin{array}{l}\text { Number } \\
\text { of methods }\end{array}$ & Search intensity & Hours searched & $\begin{array}{l}\text { Number } \\
\text { of applications }\end{array}$ & $\begin{array}{l}\text { Number } \\
\text { of interviews }\end{array}$ & Job (yes) \\
\hline & Model $1^{a}$ & Model $2^{\mathbf{b}}$ & Model $3^{a}$ & Model $4^{b}$ & Model $5^{\mathbf{b}}$ & Model $6^{b}$ & Model $7^{a}$ \\
\hline $\begin{array}{l}\text { Stigma conscious- } \\
\text { ness }(0-10)\end{array}$ & $\begin{array}{l}0.026^{* * *} \\
(0.008)\end{array}$ & $\begin{array}{l}0.067^{* * * *} \\
(0.019)\end{array}$ & $\begin{array}{l}0.282^{* * *} \\
(0.081)\end{array}$ & $\begin{array}{l}0.077^{*} \\
(0.034)\end{array}$ & $\begin{array}{l}0.069^{*} \\
(0.034)\end{array}$ & $\begin{array}{l}0.069 \\
(0.051)\end{array}$ & $\begin{array}{l}-0.004 \\
(0.005)\end{array}$ \\
\hline $\begin{array}{l}\text { Sample (R: popula- } \\
\text { tion sample) }\end{array}$ & $\begin{array}{l}0.052 \\
(0.059)\end{array}$ & $\begin{array}{l}0.096 \\
(0.149)\end{array}$ & $\begin{array}{l}0.620 \\
(0.608)\end{array}$ & $\begin{array}{l}0.380^{\#} \\
(0.213)\end{array}$ & $\begin{array}{l}0.152 \\
(0.267)\end{array}$ & $\begin{array}{l}1.024^{*} \\
(0.501)\end{array}$ & $\begin{array}{l}0.019 \\
(0.034)\end{array}$ \\
\hline \multicolumn{8}{|c|}{ Employment status at wave 6 (R: employed) } \\
\hline Unemployed & $\begin{array}{l}-0.038 \\
(0.043)\end{array}$ & $\begin{array}{l}-0.006 \\
(0.094)\end{array}$ & $\begin{array}{l}0.003 \\
(0.457)\end{array}$ & $\begin{array}{l}0.168 \\
(0.147)\end{array}$ & $\begin{array}{l}-0.037 \\
(0.179)\end{array}$ & $\begin{array}{l}-0.266 \\
(0.272)\end{array}$ & $\begin{array}{l}-0.147^{* * * *} \\
(0.032)\end{array}$ \\
\hline $\begin{array}{l}\text { Out of the labour } \\
\text { force }\end{array}$ & $\begin{array}{l}0.070 \\
(0.136)\end{array}$ & $\begin{array}{l}-0.072 \\
(0.298)\end{array}$ & $\begin{array}{l}0.740 \\
(1.718)\end{array}$ & $\begin{array}{l}0.215 \\
(0.436)\end{array}$ & $\begin{array}{l}-0.913^{\#} \\
(0.489)\end{array}$ & $\begin{array}{l}-0.521 \\
(0.584)\end{array}$ & $\begin{array}{l}-0.011 \\
(0.110)\end{array}$ \\
\hline Male & $\begin{array}{l}0.047^{\#} \\
(0.028)\end{array}$ & $\begin{array}{l}0.098 \\
(0.067)\end{array}$ & $\begin{array}{l}0.551^{\#} \\
(0.309)\end{array}$ & $\begin{array}{l}0.283^{* *} \\
(0.107)\end{array}$ & $\begin{array}{l}0.156 \\
(0.126)\end{array}$ & $\begin{array}{l}0.587^{* *} \\
(0.189)\end{array}$ & $\begin{array}{l}0.006 \\
(0.018)\end{array}$ \\
\hline $\begin{array}{l}\text { Unemployment } \\
\text { duration (years) }\end{array}$ & $\begin{array}{l}-0.010^{* * *} \\
(0.003)\end{array}$ & $\begin{array}{l}-0.034^{* * *} \\
(0.009)\end{array}$ & $\begin{array}{l}-0.114^{* * *} \\
(0.032)\end{array}$ & $\begin{array}{l}-0.063^{* * *} \\
(0.013)\end{array}$ & $\begin{array}{l}-0.061^{* * *} \\
(0.016)\end{array}$ & $\begin{array}{l}-0.151^{* * *} \\
(0.032)\end{array}$ & $\begin{array}{l}-0.006^{* * *} \\
(0.002)\end{array}$ \\
\hline $\begin{array}{c}\text { Number of previ- } \\
\text { ous unemploy- } \\
\text { ment episodes }\end{array}$ & $\begin{array}{l}0.019 \\
(0.018)\end{array}$ & $\begin{array}{l}0.060 \\
(0.041)\end{array}$ & $\begin{array}{l}0.304 \\
(0.212)\end{array}$ & $\begin{array}{l}0.017 \\
(0.063)\end{array}$ & $\begin{array}{l}0.049 \\
(0.071)\end{array}$ & $\begin{array}{l}0.022 \\
(0.129)\end{array}$ & $\begin{array}{l}0.044^{* * *} \\
(0.013)\end{array}$ \\
\hline Age & $\begin{array}{l}0.041^{* * *} \\
(0.009)\end{array}$ & $\begin{array}{l}0.122^{* * *} \\
(0.025)\end{array}$ & $\begin{array}{l}0.444^{* * *} \\
(0.101)\end{array}$ & $\begin{array}{l}0.171^{* * *} \\
(0.037)\end{array}$ & $\begin{array}{l}0.111^{* *} \\
(0.042)\end{array}$ & $\begin{array}{l}0.114^{\#} \\
(0.063)\end{array}$ & $\begin{array}{l}0.003 \\
(0.006)\end{array}$ \\
\hline $\mathrm{Age}^{2}$ & $\begin{array}{l}-0.001^{* * *} \\
(0.000)\end{array}$ & $\begin{array}{l}-0.002^{* * *} \\
(0.000)\end{array}$ & $\begin{array}{l}-0.006^{* * *} \\
(0.001)\end{array}$ & $\begin{array}{l}-0.002^{* * *} \\
(0.000)\end{array}$ & $\begin{array}{l}-0.002^{* *} \\
(0.000)\end{array}$ & $\begin{array}{l}-0.002^{*} \\
(0.001)\end{array}$ & $\begin{array}{l}-0.000 \\
(0.000)\end{array}$ \\
\hline $\begin{array}{l}\text { Migration back- } \\
\text { ground }\end{array}$ & $\begin{array}{l}-0.057^{\#} \\
(0.035)\end{array}$ & $\begin{array}{l}-0.074 \\
(0.086)\end{array}$ & $\begin{array}{l}-0.391 \\
(0.381)\end{array}$ & $\begin{array}{l}0.221^{\#} \\
(0.129)\end{array}$ & $\begin{array}{l}0.059 \\
(0.149)\end{array}$ & $\begin{array}{l}0.148 \\
(0.196)\end{array}$ & $\begin{array}{l}0.041^{\#} \\
(0.023)\end{array}$ \\
\hline \multicolumn{8}{|c|}{ Education (R: elementary) (CASMIN) } \\
\hline Secondary & $\begin{array}{l}0.035 \\
(0.034)\end{array}$ & $\begin{array}{l}0.192^{*} \\
(0.078)\end{array}$ & $\begin{array}{l}0.914^{*} \\
(0.379)\end{array}$ & $\begin{array}{l}0.165 \\
(0.130)\end{array}$ & $\begin{array}{l}-0.152 \\
(0.140)\end{array}$ & $\begin{array}{l}-0.212 \\
(0.225)\end{array}$ & $\begin{array}{l}0.009 \\
(0.021)\end{array}$ \\
\hline Maturity & $\begin{array}{l}0.020 \\
(0.048)\end{array}$ & $\begin{array}{l}0.145 \\
(0.115)\end{array}$ & $\begin{array}{l}0.489 \\
(0.527)\end{array}$ & $\begin{array}{l}0.174 \\
(0.184)\end{array}$ & $\begin{array}{l}-0.013 \\
(0.223)\end{array}$ & $\begin{array}{l}0.069 \\
(0.267)\end{array}$ & $\begin{array}{l}-0.000 \\
(0.032)\end{array}$ \\
\hline Tertiary & $\begin{array}{l}0.128^{* *} \\
(0.048)\end{array}$ & $\begin{array}{l}0.313^{* *} \\
(0.106)\end{array}$ & $\begin{array}{l}1.497^{* *} \\
(0.536)\end{array}$ & $\begin{array}{l}0.273^{\#} \\
(0.153)\end{array}$ & $\begin{array}{l}0.229 \\
(0.210)\end{array}$ & $\begin{array}{l}-0.263 \\
(0.259)\end{array}$ & $\begin{array}{l}0.094^{* *} \\
(0.034)\end{array}$ \\
\hline Married & $\begin{array}{l}-0.053 \\
(0.038)\end{array}$ & $\begin{array}{l}-0.107 \\
(0.096)\end{array}$ & $\begin{array}{l}-0.413 \\
(0.408)\end{array}$ & $\begin{array}{l}-0.188 \\
(0.155)\end{array}$ & $\begin{array}{l}-0.184 \\
(0.176)\end{array}$ & $\begin{array}{l}0.013 \\
(0.227)\end{array}$ & $\begin{array}{l}-0.005 \\
(0.024)\end{array}$ \\
\hline Household size & $\begin{array}{l}-0.006 \\
(0.015)\end{array}$ & $\begin{array}{l}-0.039 \\
(0.041)\end{array}$ & $\begin{array}{l}-0.187 \\
(0.168)\end{array}$ & $\begin{array}{l}-0.108^{\#} \\
(0.059)\end{array}$ & $\begin{array}{l}-0.042 \\
(0.087)\end{array}$ & $\begin{array}{l}0.045 \\
(0.090)\end{array}$ & $\begin{array}{l}-0.007 \\
(0.011)\end{array}$ \\
\hline $\begin{array}{l}\text { Household income } \\
\qquad \text { (in } 100 €)\end{array}$ & $\begin{array}{l}0.006^{*} \\
(0.003)\end{array}$ & $\begin{array}{l}0.016^{*} \\
(0.008)\end{array}$ & $\begin{array}{l}0.070^{*} \\
(0.032)\end{array}$ & $\begin{array}{l}0.027^{*} \\
(0.011)\end{array}$ & $\begin{array}{l}0.012 \\
(0.016)\end{array}$ & $\begin{array}{l}-0.017 \\
(0.018)\end{array}$ & $\begin{array}{l}0.006^{*} \\
(0.002)\end{array}$ \\
\hline West Germany & $\begin{array}{l}-0.058^{\#} \\
(0.031)\end{array}$ & $\begin{array}{l}-0.204^{* *} \\
(0.074)\end{array}$ & $\begin{array}{l}-0.781^{*} \\
(0.334)\end{array}$ & $\begin{array}{l}-0.396^{* *} \\
(0.124)\end{array}$ & $\begin{array}{l}-0.191 \\
(0.134)\end{array}$ & $\begin{array}{l}0.048 \\
(0.212)\end{array}$ & $\begin{array}{l}-0.010 \\
(0.019)\end{array}$ \\
\hline Constant & $\begin{array}{l}-0.322 \\
(0.206)\end{array}$ & $\begin{array}{l}-2.036^{* * *} \\
(0.538)\end{array}$ & $\begin{array}{l}-5.268^{*} \\
(2.207)\end{array}$ & $\begin{array}{l}-2.733^{* * *} \\
(0.818)\end{array}$ & $\begin{array}{l}-0.585 \\
(0.927)\end{array}$ & $\begin{array}{l}-3.701^{* *} \\
(1.421)\end{array}$ & $\begin{array}{l}0.209 \\
(0.141)\end{array}$ \\
\hline Observations & 1278 & 1278 & 1278 & 1278 & 1278 & 1278 & 1573 \\
\hline
\end{tabular}

Weighted to account for panel attrition from waves 7 to 8 ; multiple imputations were performed by chained equations to account for missing data values (40 imputations, burn in period of 30 iterations). Goodness-of-fit tests are not available after multiple imputations in STATA (see STATA multiple imputation reference manual release 13, p. 79)

Outcome variables Models 1 to 5: job search activity (yes/no); number of job search methods; job search intensity; number of hours spent searching; number of applications

Outcome variables Models 6 and 7: number of job interviews; employed (yes/no) Robust standard errors in parentheses; ${ }^{* *} \mathrm{p}<0.001,{ }^{* *} \mathrm{p}<0.01,{ }^{*} \mathrm{p}<0.05,{ }^{*} \mathrm{p}<0.10$ Estimation methods: ${ }^{\mathrm{a}}$ linear regression, ${ }^{\mathrm{b}}$ negative binomial count data regression 
Our empirical analysis based on several different indicators of job search effort finds empirical support for Hypothesis 5b, i.e., stigma-conscious unemployed individuals increase their job search effort.

Turning to Hypothesis 6, we have two different indicators of an unemployed individual's job search success. The first indicator focusses on the number of job interviews during the last 4 weeks of those still unemployed (Model 6). Here, the association with stigma consciousness is positive but the coefficient is small and statistically insignificant. Considering Model 7 in which the dependent variable is actual re-employment, we find that stigma consciousness is slightly negatively related to re-employment probability, but again, this effect is not statistically significant.

\subsection{Robustness checks}

Here, we report some robustness checks and tests of potential alternative explanations for our empirical results.

A first alternative explanation concerns the relationship between stigma consciousness and job-search effort. We cannot exclude the possibility that the level of job search effort could lead to high stigma consciousness and not the other way around. For example, we might assume that those who are starting their job search with above average job search effort are more prone to interpret their experience of continued unemployment as the result of stigmatization. In both cases, a positive regression coefficient of job search effort on stigma consciousness will arise in cross-sectional data. However, if the regression coefficients reflect such differences in job search effort at the beginning of the unemployment spell, the positive relationship should disappear once these initial differences are accounted for. Thus, as a robustness check, we measure the job search effort at the start of the unemployment episode. Specifically, we use the job search effort from the start of the unemployment spell or if observations remain censored because the respondents entered the panel survey during unemployment, job search effort from the first observed wave. ${ }^{6}$ The underlying idea here is that job search effort during the early stage of the unemployment spell is not influenced by stigma consciousness because it is defined as the expectation of being subjected to negative stereotypes during the job search. To the degree that these expectations

\footnotetext{
${ }^{6}$ For example, if an individual entered unemployment during wave 4 and is still observed as unemployed during wave 7 (our main sample selection criterion), the indicator measures job search effort during wave 4 . If the individual entered the panel survey during wave 5 as unemployed and was unemployed at least until wave 7 , the job search effort is measured at wave 5 . If the individual was employed before wave 7 , the job search effort on the job is measured at wave 6.
}

are based on actual experiences, measuring job search behaviour at the beginning of the job search suggests that only minimal experiences have been gathered and that the level of job search effort should still be relatively independent of such negative experiences.

Unfortunately, information regarding the initial job search effort is only available for active job search, number of job search methods and number of job interviews. However, at least for these outcomes, we can perform a robustness check by including the respective initial values as additional control variables. We extend this procedure to the analysis of life and health satisfaction and we control for the initial value at the first observed wave in unemployment, too. As shown in Appendix, Table 8 , including the initial levels of the dependent variables reduces the size of the coefficients of stigma consciousness in all cases, but the basic conclusions remain unchanged. The coefficients tend to be smaller but are still positive and statistically significant. However, using past values of the dependent variable (so-called lags) has been criticized not only in the context of panel data (Nickell 1981) but also, more recently, in pooled crosssectional data analysis by Vaisey and Miles (2017). Thus, this strategy might not be able to remove the bias entirely. ${ }^{\text {? }}$

A second alternative explanation is concerned with whether increasing the job search effort is really based on the autonomous decision of the unemployed as suggested by our theoretical framework. In contrast, as posited by self-determination theory (Ryan and Deci 2000), increased effort can also be the result of externally controlled behaviour. Following Hirseland and Ramos Lobato (2014), we might assume that the increased job search effort of those unemployed experiencing high

\footnotetext{
${ }^{7}$ In our application, lagging the dependent variable could pose a problem if in technical terms, large reverse causality bias and large bias due to unobserved confounders coincide. According to results from a Monte Carlo simulation by Vaisey and Miles (2017), if bias due to unobserved confounders is low to medium, the results of a regression with and without lagged dependent variables can be considered the lower and upper bounds, e.g., for the dependent variable "active job search (yes)", the upper bound is 0.026 (Table 2, Model 1) and the lower bound is 0.014 (see Table 8, Model 3). However, we emphasize that there is an important difference between our strategy and the strategy criticized by Vaisey and Miles (2017). We do not simply lag the dependent variable for one period, which was the approach used by Vaisey and Mills (2017). In that case, we would still use a value of the dependent variable (job search effort) that has potentially been influenced by prior values of the focal independent variable (stigma consciousness). Instead, we aim to control for the initial (or at least the earliest observed) value of job search effort. Thus, we aim to measure the dependent variable at a point in its history when it is still "uncontaminated" by the focal independent variable. In the simulated data reported by Vaisey and Miles (2017), there is never such a point in the common history of the dependent and independent variables, which may explain why lagging does not work as intended. To clarify this distinction, we refer to "initial values" instead of "lags" of the dependent variables (see Table 8 in Appendix).
} 
stigma consciousness could be the result of their attempts to comply with the demands placed upon them by case workers at local unemployment offices. To "activate" the unemployed, case workers often monitor their job search effort and sanction those who do not comply with what in Germany is called "Mitwirkungspflicht" (duty to cooperate). If this monitoring is successful such that it leads to increased job search efforts but simultaneously makes the unemployed feel depreciated and stereotyped as the "lazy unemployed", high stigma consciousness could arise as a by-product of such monitoring practices. Consequently, the positive effect of stigma consciousness could be spurious and solely based on the level of monitoring through unemployment offices and their case workers as a common cause. To test this alternative explanation, we included information on whether an integration agreement was signed between the unemployed and the case worker as an additional covariate. Furthermore, we included a factor score measuring the self-perceived quality of the unemployeds' experiences with the job center and their staff members. The factor sore was derived from a factor analyses on items of a respective items set. ${ }^{8}$ We found that the inclusion of these variables did not substantially change the results (see Tables 9 and 10 in Appendix).

Third, a further alternative explanation, especially for the results concerning job search effort, is social desirability bias. Social desirability bias refers to survey respondents' tendency to adapt their answers towards what they perceive to be the social norm. To explain the positive association between stigma consciousness and effort, social desirability bias must upward bias the reporting of both job search effort and stigma consciousness, net of all covariates, such as age, gender and education. Clearly, there is a danger that individuals tend to overstate their job search effort in an interview situation because given the public debate regarding the lazy unemployed, high effort to end unemployment is normatively more acceptable than low effort. However, regarding stigma consciousness, the nature of social stigma is that it is rather hidden in social interactions than overemphasized. Therefore, social desirability bias is more likely to lead to a downward bias in reporting the level of stigma experienced during unemployment. ${ }^{9}$ Overall, this logic argues against social desirability bias as an alternative explanation.

Fourth, in accordance with our theoretical framework, many of our dependent variables are based on selfassessment and measure respondent's perceptions, e.g., of being subjected to stereotypes or of their labour market chances. Therefore, personality traits such as self-efficacy or the "Big 5 " personality traits might be a common cause for the negative relationship of stigma consciousness and subjective well-being and/or with the positive association with job search effort. Therefore, in a further robustness check, we conducted our analysis controlling for these traits. Self-efficacy is defined as generalized selfefficacy and was measured as sum score obtained from five items ${ }^{10}$ measured in wave 7 . These items focus on the personal assessment of one's own competences to deal with difficulties and barriers in everyday life (Schwarzer and Jerusalem 1995, 1999) and were used as sum indices. Further personality traits were measured using the 21-item version of the Big Five Inventory (BFI-K) which covers rather broad personality dimensions extraversion, agreeableness, conscientiousness, neuroticism and openness to experience (Rammstedt and John 2005). Those traits are only available in wave 5 and for those respondents in our sample who did not already participate in that wave, values had to be multiply imputed. We found that even if some of the personality traits are significantly correlated with some outcomes, including self-efficacy or the "Big 5" personality traits does not substantially change the results. Whereas the coefficients of stigma consciousness for well-being and health become smaller, those for job-search effort even slightly increase (see Tables 11 and 12 in Appendix).

\footnotetext{
${ }^{8}$ How far do the following statements apply to your personal experience with the Job centre and their staff members? Please tell me whether these statements "Apply completely", "Tend to apply", "Tend not to apply" or "Do not apply at all". (A) The staff dictate too much what I am to do; (B) They really want to help me there; $(C)$ I expect that my situation will improve through the counselling; (D) They support me in finding a job again; (E) Only demands are put forward by them, but I don't get any support; (F) I trust the staff; (G) My ideas are taken into consideration in counselling; (v) The staff members are friendly and helpful to me; One item (C) had to be excluded from the scale because of positive and negative correlations with the other items, two items (A and E) were reversed so that higher values indicate a more positive experience. The scale proved to be one-dimensional and is sufficiently reliable (Cronbach's alpha 0.85). The scale was only presented to those unemployed that were actually registered with the job center, i.e., recipients of welfare benefits, thus the number of observations are slightly lower compared to the main analysis.
}

\footnotetext{
${ }^{9}$ Since the PASS is a dual mode survey, one way to test for social desirability bias is to determine whether both job-search effort and stigma consciousness are higher among those engaged in face-to-face interviews compared to those engaged in telephone interviews (under the assumption that social desirable answers are more common face-to-face). However, we observe lower instead of higher job-search effort in face-to-face interviews, which is not consistent with social desirability bias.

${ }^{10}$ Whenever unexpected difficulties or problems show up, there are different ways of reacting to that. We grouped some opinions about that topic here. Please tell me, whether to you those opinions "Apply completely", "Tend to apply", "Tend not to apply" or "Do not apply at all". (A) I have a solution for every problem. (B) Even when things happen surprisingly, I believe that I can cope with them. (C) I have no difficulties in achieving my aims. (D) I always know how to act in unforeseeable situations. (E) I can always solve difficult problems if I try to.
} 
Finally, the Appendix also documents that the results without multiple imputation are, except for higher standard errors, similar to those after multiple imputation (see Tables 13 and 14 in Appendix).

\subsection{Limitations}

An important caveat of our analysis is that our results might only apply to the German context and need not necessarily extend to countries with different systems of social security or welfare traditions. In general, Germany is assumed to be characterized by a social security system that has a strong focus on status maintenance. For example, Paugam and Russell (2000) argue that due to the high importance of employment for social status in Germany, unemployment is likely to lead to social stigma. Within the German context, our analytical sample is characterized by a high share of long-term unemployed, mostly recipients of welfare benefits. In Germany, unemployment insurance benefit receipt is limited to 12 months for the general population and 24 month for workers 55 years or older. After the insurance benefits expire, the unemployed can receive means-tested basic income support. Basic income support consists of a flat rate, and the unemployed are only eligible for support if their household income is below a certain threshold. However, our indicator of stigma consciousness does not focus on the stigma of welfare receipt but on the general stigma of unemployment. Therefore, we cannot answer the question regarding whether a measure of stigma consciousness focusing more on welfare state dependency could lead to different results. In addition, due to our analytical sample restrictions, we focus on unsuccessful job searches, increasing the tendency to over represent the long-term unemployed. However, the long-term unemployed should be subject to stronger stigma than the short-term unemployed, and unsuccessful job searches should be more likely to lead to passivity. Therefore, notably, even in this analytical sample (average unemployment duration in wave 6 is slightly over 5 years), the association with job search effort is positive.

A second limitation concerns the results on actual re-employment. The results for job search effort and the number of job interviews on the one hand and reemployment chances on the other hand are observed on systematically divergent populations (the former is only observed among those who did not find re-employment), the interpretation that increased job search effort does not increase re-employment chances can thus be challenged. In addition for re-employment, a duration analysis might have been the more informative and appropriate method. However, even if our focal independent variable stigma consciousness is in principle time-varying, it was measured in PASS at only one point in time. This point in time is different for all respondents with respect to their previous unemployment duration. Given that unemployment duration influences stigma consciousness, it is crucial for our analysis to control for this variable. In a duration analysis, unemployment duration would already be the dependent variable, therefore controlling for elapsed unemployment duration until the stigma consciousness was measured would induce endogeneity. Therefore, we rely on the simpler logistic regression model, where we can control for unemployment duration. We acknowledge, however, that this discards a lot of information and is only a workaround.

\section{Discussion and conclusion}

An important strand of literature in labour market research is concerned with the effect of unemployment stigma on re-employment chances. This literature shows that the unemployed are stigmatized in the sense that they face serious disadvantages on the labour market, irrespective of their actual motivation, skills and behaviour and that unemployment can create a vicious cycle where unemployment begets further unemployment. In contrast, literature on the behaviour of the unemployed themselves is scarce and prone to assume that the typical reaction of the unemployed to being stigmatized is passivity and withdrawal behaviour.

Our paper is the first to present an empirical test of how stigma consciousness relates to job search attitudes and behaviour. We tested several hypotheses and interpreted the empirical evidence as follows. First, we corroborated the results of other studies showing that being stigmatized has negative consequences on individual well-being and health. Those who rated higher on the stigma consciousness scale also showed significantly lower life and health satisfaction. Based on expectancyvalue theory, we found that being subjected to the social stigma of unemployment leads the unemployed to have lower expectations of successfully leaving unemployment. In contrast, the value of employment increases most likely because re-employment is an effective way to free the unemployed from unemployment stigma.

While the literature on unemployment suggests discouragement or withdrawal among the unemployed, we posit two possible reactions of the unemployed towards perceiving themselves stigmatized with respect 
to job search behaviour. If the low expected employment chances dominate the stigmatized's behaviour, these individuals should decrease their job search effort. In contrast, if a higher value of employment dominates, the stigmatized should actually increase their job search effort. By presenting empirical evidence from several different indicators of job search effort, we found that high values of stigma consciousness were associated with more rather than less effort, e.g., in terms of engaging in an active job search, the number of hours spent searching or the number of job applications. We interpret this as evidence that the stigmatized unemployed are not characterized by passiveness or learned helplessness as the literature sometimes suggests. In contrast, we interpret this finding as evidence that the stigmatized suffer from their experience of joblessness even more than the average unemployed and aim to leave unemployment to change their social status and eliminate the social stigma. However, for individuals subjected to unemployment stigma, such increased effort does not lead to better actual reemployment chances. Despite its positive association with job search effort, we found no statistically significant association between stigma consciousness and the number of job-interviews or even re-employment probability.

Overall, we interpret our results as evidence that those who experience unemployment stigma during their job search suffer more from their experience of joblessness but do not tend to react with withdrawal and passivity. These individuals do not quit the job search and instead increase their effort by utilizing more methods to search for a job, spend more hours searching and send more job applications to potential employers. However, no empirical evidence supports that this increased effort helps the unemployed improve their situation by leaving unemployment. This result is in line with Gielen and van Ours (2014), who find that even if the unhappy unemployed search more actively for a job, it does not impact their unemployment duration. The results are also in line with Hohmeyer and Wolff (2018) who find that One-Euro-Job announcements increase job search effort but does not lead to higher employment probability.

Because we mainly rely on cross-sectional data, alternative explanations of the observed pattern of associations are possible. For example, high stigma consciousness could instead be a reaction of high-effort job seekers who become frustrated by the absence of re-employment success. Furthermore, pressure from employment offices might be a common cause of both high stigma consciousness and high job search effort. We attempted to test these alternative explanations as much as possible and found no evidence supporting these explanations over our own. However, we must acknowledge that these alternative explanations cannot be entirely dismissed given that there seems to be no bulletproof solution, especially regarding reverse causality, which is particularly true for cross-sectional data but in many regards also extends to longitudinal data (see e.g., Vaisey and Miles 2017). With longitudinal data, more sophisticated methods are available (cf., Leszczensky and Wolbring 2018), but these methods are also not without their own problems. Therefore, further research is needed to corroborate our results. Such research should preferably be based on longitudinal data that includes the measurement of unemployment stigma at several points in time, including the beginning of unemployment. To gain such data, refining the existing scale could be worth the effort to obtain a shorter scale that is more easily incorporated into panel surveys to measure unemployment stigma.

\begin{abstract}
Acknowledgements
We thank the guest editor Katrin Auspurg, two anonymous reviewers, Katrin Hohmeyer and Jens Stegmaier for the helpful and constructive comments. Previous versions of the manuscript have also profited from comments by the participants of the Second PASS user conference in Nürnberg and the participants of the Session of the Sektion 'Sozialpolitik' at the 2018 DGS Kongress in Göttingen, especially Sigrid Betzelt and Carolin Freier. We also thank Huyen Nguyen Ngoc and Luca Reinold for help with preparing the manuscript.
\end{abstract}

Authors' contributions

All authors read and approved the final manuscript.

Funding

Monika Jungbauer-Gans received funding for this article from the Deutsche Forschungsgemeinschaft under grant DFG JU 414/15-1.

\section{Availability of data and materials}

The datasets analysed in the current study are available in the Forschungsdatenzentrum der Bundesagentur für Arbeit (BA) im Institut für Arbeitsmarktund Berufsforschung (IAB), https://fdz.iab.de/de/FDZ_Individual_Data/PASS. aspx.

\section{Competing interests}

The authors declare that they have no significant competing financial, professional, or personal interests that might have influenced the performance or presentation of the work described in this article.

\section{Author details}

${ }^{1}$ Institute for Employment Research (IAB), Regensburger Str. 104,

90478 Nuremberg, Germany. ${ }^{2}$ Friedrich-Alexander University Erlangen-Nürnberg (FAU), Erlangen, Germany. ${ }^{3}$ German Centre of Higher Education Research and Science Studies (DZHW), Hannover, Germany. 


\section{Appendix}

See Tables 3, 4, 5, 6, 7, 8, 9, 10, 11, 12, 13 and 14 .

\section{Table 3 Items of the stigma consciousness sum score}

\begin{tabular}{|c|c|c|}
\hline & $\begin{array}{l}\text { Cronbach's alpha } \\
\text { (up to item in row) }\end{array}$ & Mean (Std. dev.) \\
\hline $\begin{array}{l}\text { A. It is hard for me to keep relationships with employed people alive. (Es fällt mir schwer, Beziehungen zu } \\
\text { Menschen aufrecht zu erhalten, die erwerbstätig sind.) }\end{array}$ & & $1.77(0.87)$ \\
\hline B. I feel burdened because of being unemployed. (Es belastet mich persönlich, arbeitslos zu sein.) & & $3.13(0.94)$ \\
\hline $\begin{array}{l}\text { C. There are situations in everyday life in which I become aware that life is more difficult for someone unem- } \\
\text { ployed than for employed people. (Es gibt Situationen im Alltag, in denen mir bewusst wird, dass es für } \\
\text { Arbeitslose schwieriger ist als für Erwerbstätige.) }\end{array}$ & & $3.35(0.80)$ \\
\hline $\begin{array}{l}\text { D. I think that most people have more prejudices against the unemployed than they would openly admit. (Ich } \\
\text { denke, dass die meisten Menschen mehr Vorurteile über Arbeitslose haben als sie offen sagen. }\end{array}$ & & $3.37(0.73)$ \\
\hline $\begin{array}{l}\text { E. I feel more obliged to other unemployed people than to employed people. (Ich fühle mich eher anderen } \\
\text { arbeitslosen Personen verbunden, als Personen, die erwerbstätig sind.) }\end{array}$ & & $1.93(0.88)$ \\
\hline $\begin{array}{l}\text { F. Prejudices against the unemployed affect me personally. (Von Vorurteilen gegenüber Arbeitslosen fühle ich } \\
\text { mich persönlich betroffen.) }\end{array}$ & & $2.39(1.03)$ \\
\hline $\begin{array}{l}\text { G. In certain situations I try to hide that I am unemployed. (In bestimmten Situationen bemühe ich mich zu } \\
\text { verheimlichen, dass ich arbeitslos bin.) }\end{array}$ & & $1.96(1.02)$ \\
\hline $\begin{array}{l}\text { H. I try to avoid situations where prejudice or discrimination against the unemployed could occur. (Ich ver- } \\
\text { suche Situationen zu vermeiden, in denen es zu Vorurteilen oder Benachteiligungen gegenüber Arbeit- } \\
\text { slosen kommen könnte.) }\end{array}$ & 0.7310 & $2.30(1.04)$ \\
\hline $\begin{array}{l}\text { Excluded: I. I am trying to find a job as quickly as possible. (Ich selbst versuche, so schnell es geht, wieder } \\
\text { einen Arbeitsplatz zu bekommen.) }\end{array}$ & 0.7129 & $3.28(0.97)$ \\
\hline
\end{tabular}

Original scale for individual items: (1) agree (2) rather agree (3) rather disagree (4) disagree; scales for all items except I) were reversed so that higher values indicate higher stigmatization. German original version in parentheses, English version is official translation provided by PASS

\section{Table 4 Overview of questionnaire items covering job search efforts}

\begin{tabular}{|c|c|}
\hline Question & Answer \\
\hline $\begin{array}{l}\text { Now we would like to know what you have done in the past } 4 \text { weeks in order to find a job. From where have you gathered } \\
\text { information on jobs during the past } 4 \text { weeks? Have you... } \\
\text { A. Looked through job advertisements in the newspaper? PASS_-persons' } \\
\text { B. Looked through the employment agencies' online job market? } \\
\text { C. Searched other internet sources? } \\
\text { D. Asked family and friends? } \\
\text { E. Got information from the placement officer at the employment agency? } \\
\text { F. Got information from a private job placement service? } \\
\text { G. Other, namely (open): }\end{array}$ & Yes/no \\
\hline And how often did you do this in the last 4 weeks? "daily", "several times a week", "weekly" or "less often"? & $\begin{array}{l}1 \text { Daily } \\
2 \text { Several times a week } \\
3 \text { Weekly } \\
4 \text { Less often }\end{array}$ \\
\hline Please continue to think about the last 4 weeks. How many hours did you spend on average each week looking for jobs? & 3-digit open answer \\
\hline $\begin{array}{l}\text { There are after all other ways of looking for a job } \\
\text { I will now read out various activities to you. Please tell me, whether you have performed these activities during the past } \\
4 \text { weeks, and if so, how often? } \\
\text { A. Replied to job advertisements? } \\
\text { B. Placed an "employment wanted" advertisement with the newspaper? } \\
\text { C. Asked for a job at the company itself? } \\
\text { D. Submitted your application even though no job opening had been advertised? }\end{array}$ & 3-digit open answer \\
\hline
\end{tabular}

English version is official translation provided by PASS 
Table 5 Descriptive statistics (before multiple imputation)

\begin{tabular}{|c|c|c|c|c|c|}
\hline Variable & Obs. & Mean & Std. dev. & Min. & Max. \\
\hline \multicolumn{6}{|l|}{ Outcome variables } \\
\hline Life satisfaction & 1276 & 5.77 & 2.08 & 0 & 10 \\
\hline Health satisfaction & 1277 & 5.42 & 2.59 & 0 & 10 \\
\hline Self-assessed employment chances & 1263 & 1.83 & 0.93 & 1 & 4 \\
\hline Value of employment & 1269 & -0.04 & 1.09 & -3.50 & 1.39 \\
\hline Active job search & 1278 & 0.52 & & 0 & 1 \\
\hline Number of job search methods & 1278 & 1.75 & 1.96 & 0 & 7 \\
\hline Search intensity & 1278 & 4.77 & 5.51 & 0 & 24 \\
\hline Hours searching & 1269 & 3.21 & 6.16 & 0 & 70 \\
\hline Number of applications & 1268 & 3.60 & 7.77 & 0 & 94 \\
\hline Number of interviews & 1276 & 0.27 & 0.98 & 0 & 15 \\
\hline Found job & 1573 & 0.14 & & 0 & 1 \\
\hline \multicolumn{6}{|l|}{ Focal independent variable } \\
\hline Stigma consciousness & 1278 & 5.12 & 1.81 & 0 & 10 \\
\hline \multicolumn{6}{|l|}{ Independent variables } \\
\hline Sample (1, if welfare benefit sample) & 1278 & 0.94 & & 0 & 1 \\
\hline Employment status preceding wave & 1082 & & & & \\
\hline Employed & & 0.15 & & 0 & 1 \\
\hline Unemployed & & 0.84 & & 0 & 1 \\
\hline Out of the labour force & & 0.01 & & 0 & 1 \\
\hline Male & 1278 & 0.56 & & 0 & 1 \\
\hline Unemployment duration (in years) & 1265 & 5.05 & 4.86 & 0.08 & 43.25 \\
\hline Number of previous unemployment episodes & 1265 & 1.41 & 0.87 & 1 & 7 \\
\hline Age & 1278 & 46.46 & 11.91 & 18 & 64 \\
\hline Migration background & 1243 & 0.24 & & 0 & 1 \\
\hline Education (CASMIN) & 1276 & & & & \\
\hline Elementary & & 0.50 & & 0 & 1 \\
\hline Secondary & & 0.30 & & 0 & 1 \\
\hline Maturity & & 0.10 & & 0 & 1 \\
\hline Tertiary & & 0.10 & & 0 & 1 \\
\hline Married & 1273 & 0.26 & & 0 & 1 \\
\hline Household size & 1278 & 2.00 & 1.29 & 1 & 9 \\
\hline Household income (in $100 €$ ) & 1273 & 10.56 & 6.38 & 0 & 90 \\
\hline West Germany & 1278 & 0.59 & & 0 & 1 \\
\hline Integration agreement & 667 & & & & \\
\hline Agreement signed & & 0.78 & & 0 & 1 \\
\hline Agreement not signed & & 0.16 & & 0 & 1 \\
\hline Does not apply (not sgb II) & & 0.06 & & 0 & 1 \\
\hline Experiences with job center (negative to positive) & 864 & -0.02 & 1.01 & -1.94 & 2.19 \\
\hline Self-efficacy & 1278 & 10.95 & 2.70 & 2 & 20 \\
\hline \multicolumn{6}{|l|}{ Big five personality traits } \\
\hline Extraversion & 873 & 13.37 & 3.53 & 3 & 20 \\
\hline Agreeableness & 872 & 11.40 & 2.89 & 4 & 20 \\
\hline Conscientiousness & 872 & 14.27 & 1.95 & 7 & 20 \\
\hline Neuroticism & 872 & 11.10 & 2.03 & 5 & 17 \\
\hline Openness to experience & 872 & 18.35 & 3.76 & 7 & 25 \\
\hline Initial life satisfaction & 1077 & 5.63 & 2.28 & 0 & 10 \\
\hline Initial health & 1077 & 5.44 & 2.61 & 0 & 10 \\
\hline Initial active job search (yes) & 1074 & 0.52 & & 0 & 1 \\
\hline Initial number of job search methods & 1058 & 1.43 & 1.93 & 0 & 7 \\
\hline Initial number of job interviews & 1058 & 0.20 & 1.16 & 0 & 28 \\
\hline
\end{tabular}

Descriptive statistics are reported for the sample of potential job seekers except for the variable "Found job" 
Table 6 Effects of unemployment stigma on subjective well-being (H1), health (H2), re-employment expectations (H3) and value placed on employment (H4) without controlling for unemployment duration

\begin{tabular}{|c|c|c|c|c|}
\hline & $\begin{array}{l}\text { H1: Subjective well-being } \\
\text { Model } 1^{\text {a }}\end{array}$ & $\begin{array}{l}\text { H2: Health } \\
\text { Model } 2^{\mathrm{a}}\end{array}$ & $\begin{array}{l}\text { H3: Expectations } \\
\text { Model } 3^{\mathrm{a}}\end{array}$ & $\begin{array}{l}\text { H4: Value } \\
\text { Model } 4^{\mathrm{a}}\end{array}$ \\
\hline Stigma consciousness (0-10) & $\begin{array}{l}-0.318^{* * *} \\
(0.033)\end{array}$ & $\begin{array}{l}-0.204^{* * *} \\
(0.039)\end{array}$ & $\begin{array}{l}-0.051^{* * *} \\
(0.013)\end{array}$ & $\begin{array}{l}0.127^{* * *} \\
(0.017)\end{array}$ \\
\hline Sample (R: population sample) & $\begin{array}{l}-0.149 \\
(0.234)\end{array}$ & $\begin{array}{l}-0.007 \\
(0.303)\end{array}$ & $\begin{array}{l}0.041 \\
(0.099)\end{array}$ & $\begin{array}{l}0.026 \\
(0.141)\end{array}$ \\
\hline \multicolumn{5}{|c|}{ Employment status at wave 6 (R: employed) } \\
\hline Unemployed & $\begin{array}{l}-0.287 \\
(0.179)\end{array}$ & $\begin{array}{l}-0.275 \\
(0.238)\end{array}$ & $\begin{array}{l}-0.267^{* *} \\
(0.081)\end{array}$ & $\begin{array}{l}-0.201^{*} \\
(0.093)\end{array}$ \\
\hline Out of the labour force & $\begin{array}{l}-0.198 \\
(0.563)\end{array}$ & $\begin{array}{l}-0.349 \\
(0.742)\end{array}$ & $\begin{array}{l}-0.026 \\
(0.327)\end{array}$ & $\begin{array}{l}0.183 \\
(0.379)\end{array}$ \\
\hline Male & $\begin{array}{l}-0.355^{* *} \\
(0.114)\end{array}$ & $\begin{array}{l}-0.024 \\
(0.144)\end{array}$ & $\begin{array}{l}0.072 \\
(0.050)\end{array}$ & $\begin{array}{l}-0.175^{* *} \\
(0.060)\end{array}$ \\
\hline Age & $\begin{array}{l}-0.099 * * \\
(0.038)\end{array}$ & $\begin{array}{l}-0.291^{* * *} \\
(0.048)\end{array}$ & $\begin{array}{l}0.005 \\
(0.016)\end{array}$ & $\begin{array}{l}0.036^{\#} \\
(0.021)\end{array}$ \\
\hline $\mathrm{Age}^{2}$ & $\begin{array}{l}0.001^{*} \\
(0.000)\end{array}$ & $\begin{array}{l}0.003^{* * *} \\
(0.001)\end{array}$ & $\begin{array}{l}-0.000^{*} \\
(0.000)\end{array}$ & $\begin{array}{l}-0.000 \\
(0.000)\end{array}$ \\
\hline Migration background & $\begin{array}{l}0.348^{*} \\
(0.135)\end{array}$ & $\begin{array}{l}0.406^{*} \\
(0.177)\end{array}$ & $\begin{array}{l}0.084 \\
(0.064)\end{array}$ & $\begin{array}{l}0.016 \\
(0.074)\end{array}$ \\
\hline \multicolumn{5}{|c|}{ Education (R: elementary) (CASMIN) } \\
\hline Secondary & $\begin{array}{l}-0.070 \\
(0.131)\end{array}$ & $\begin{array}{l}-0.106 \\
(0.169)\end{array}$ & $\begin{array}{l}0.015 \\
(0.060)\end{array}$ & $\begin{array}{l}-0.130^{\#} \\
(0.073)\end{array}$ \\
\hline Maturity & $\begin{array}{l}-0.115 \\
(0.176)\end{array}$ & $\begin{array}{l}0.564^{*} \\
(0.224)\end{array}$ & $\begin{array}{l}0.122 \\
(0.086)\end{array}$ & $\begin{array}{l}-0.374^{* * *} \\
(0.090)\end{array}$ \\
\hline Tertiary & $\begin{array}{l}-0.155 \\
(0.208)\end{array}$ & $\begin{array}{l}0.618^{*} \\
(0.240)\end{array}$ & $\begin{array}{l}0.169^{\#} \\
(0.088)\end{array}$ & $\begin{array}{l}-0.329^{* * *} \\
(0.099)\end{array}$ \\
\hline Married & $\begin{array}{l}0.095 \\
(0.151)\end{array}$ & $\begin{array}{l}-0.103 \\
(0.188)\end{array}$ & $\begin{array}{l}-0.021 \\
(0.068)\end{array}$ & $\begin{array}{l}0.170^{*} \\
(0.084)\end{array}$ \\
\hline Household size & $\begin{array}{l}0.063 \\
(0.057)\end{array}$ & $\begin{array}{l}0.062 \\
(0.081)\end{array}$ & $\begin{array}{l}-0.043 \\
(0.029)\end{array}$ & $\begin{array}{l}0.002 \\
(0.037)\end{array}$ \\
\hline Household income & $\begin{array}{l}0.010 \\
(0.011)\end{array}$ & $\begin{array}{l}0.021 \\
(0.015)\end{array}$ & $\begin{array}{l}0.002 \\
(0.005)\end{array}$ & $\begin{array}{l}0.009 \\
(0.007)\end{array}$ \\
\hline West Germany & $\begin{array}{l}-0.003 \\
(0.124)\end{array}$ & $\begin{array}{l}-0.400^{* *} \\
(0.153)\end{array}$ & $\begin{array}{l}0.105^{\#} \\
(0.054)\end{array}$ & $\begin{array}{l}-0.079 \\
(0.065)\end{array}$ \\
\hline Constant & $\begin{array}{l}9.886^{* * * *} \\
(0.819)\end{array}$ & $\begin{array}{l}13.241^{* * *} \\
(1.092)\end{array}$ & $\begin{array}{l}2.834^{* * *} \\
(0.369)\end{array}$ & $\begin{array}{l}-1.473^{* *} \\
(0.466)\end{array}$ \\
\hline Observations & 1278 & 1278 & 1278 & 1278 \\
\hline
\end{tabular}

Weighted to account for panel attrition from wave 7 to 8 ; multiple imputation by chained equations to account for missing values data (40 imputations, burn in period of 30 iterations). Goodness-of-fit tests not available after multiple imputation in STATA (see STATA multiple imputation reference manual release 13, p. 79)

Outcome variables Models 1 to 4: Life satisfaction (11-pt. scale); health satisfaction (11-pt. scale); self-assessed re-employment chances (4-pt. scale) factor score nonmonetary motivation to work (continuous)

Robust standard errors in parentheses; ${ }^{* * *} p<0.001,{ }^{* *} p<0.01,{ }^{*} p<0.05,{ }^{*} p<0.10$

Estimation methods: ${ }^{\mathrm{a}}$ linear regression 
Table 7 Effects of unemployment stigma on job search effort (H5) and success (H6) without controlling for unemployment duration

\begin{tabular}{|c|c|c|c|c|c|c|c|}
\hline & \multicolumn{5}{|c|}{$\mathrm{H} 5 \mathrm{a} / \mathrm{b}$ : Job search effort } & \multicolumn{2}{|c|}{$\begin{array}{l}\text { H6a/b: Job search } \\
\text { success }\end{array}$} \\
\hline & $\begin{array}{l}\text { Active job search } \\
\text { (yes) }\end{array}$ & $\begin{array}{l}\text { Number } \\
\text { of methods }\end{array}$ & Search intensity & Hours searched & $\begin{array}{l}\text { Number } \\
\text { of applications }\end{array}$ & $\begin{array}{l}\text { Number } \\
\text { of interviews }\end{array}$ & Job (yes) \\
\hline & Model $1^{\mathrm{a}}$ & Model $2^{b}$ & Model $3^{a}$ & Model $4^{\mathrm{b}}$ & Model $5^{b}$ & Model $6^{b}$ & Model $7^{\mathrm{a}}$ \\
\hline $\begin{array}{l}\text { Stigma conscious- } \\
\text { ness }(0-10)\end{array}$ & $\begin{array}{l}0.024^{* *} \\
(0.008)\end{array}$ & $\begin{array}{l}0.062^{* * *} \\
(0.018)\end{array}$ & $\begin{array}{l}0.261 * * \\
(0.081)\end{array}$ & $\begin{array}{l}0.064^{\#} \\
(0.035)\end{array}$ & $\begin{array}{l}0.061^{\#} \\
(0.034)\end{array}$ & $\begin{array}{l}0.048 \\
(0.050)\end{array}$ & $\begin{array}{l}-0.005 \\
(0.005)\end{array}$ \\
\hline \multicolumn{8}{|c|}{ Employment status at wave 6 (R: employed) } \\
\hline Unemployed & $\begin{array}{l}-0.073^{\#} \\
(0.042)\end{array}$ & $\begin{array}{l}-0.125 \\
(0.091)\end{array}$ & $\begin{array}{l}-0.427 \\
(0.452)\end{array}$ & $\begin{array}{l}0.008 \\
(0.139)\end{array}$ & $\begin{array}{l}-0.223 \\
(0.171)\end{array}$ & $\begin{array}{l}-0.594^{*} \\
(0.240)\end{array}$ & $\begin{array}{l}-0.184^{* * *} \\
(0.031)\end{array}$ \\
\hline $\begin{array}{l}\text { Out of the labour } \\
\text { force }\end{array}$ & $\begin{array}{l}0.060 \\
(0.138)\end{array}$ & $\begin{array}{l}-0.122 \\
(0.299)\end{array}$ & $\begin{array}{l}0.606 \\
(1.693)\end{array}$ & $\begin{array}{l}0.215 \\
(0.441)\end{array}$ & $\begin{array}{l}-0.918^{\#} \\
(0.490)\end{array}$ & $\begin{array}{l}-0.565 \\
(0.593)\end{array}$ & $\begin{array}{l}-0.038 \\
(0.108)\end{array}$ \\
\hline Male & $\begin{array}{l}0.054^{\#} \\
(0.028)\end{array}$ & $\begin{array}{l}0.125^{\#} \\
(0.067)\end{array}$ & $\begin{array}{l}0.637^{*} \\
(0.310)\end{array}$ & $\begin{array}{l}0.313^{* *} \\
(0.108)\end{array}$ & $\begin{array}{l}0.182 \\
(0.125)\end{array}$ & $\begin{array}{l}0.697^{* * *} \\
(0.197)\end{array}$ & $\begin{array}{l}0.016 \\
(0.018)\end{array}$ \\
\hline Age & $\begin{array}{l}0.039^{* * *} \\
(0.009)\end{array}$ & $\begin{array}{l}0.116^{* * *} \\
(0.024)\end{array}$ & $\begin{array}{l}0.429^{* * *} \\
(0.100)\end{array}$ & $\begin{array}{l}0.153^{* * *} \\
(0.039)\end{array}$ & $\begin{array}{l}0.104^{*} \\
(0.041)\end{array}$ & $\begin{array}{l}0.094 \\
(0.064)\end{array}$ & $\begin{array}{l}0.004 \\
(0.006)\end{array}$ \\
\hline $\mathrm{Age}^{2}$ & $\begin{array}{l}-0.001^{* * *} \\
(0.000)\end{array}$ & $\begin{array}{l}-0.002^{* * *} \\
(0.000)\end{array}$ & $\begin{array}{l}-0.006^{* * *} \\
(0.001)\end{array}$ & $\begin{array}{l}-0.002^{* * *} \\
(0.000)\end{array}$ & $\begin{array}{l}-0.002^{* *} \\
(0.000)\end{array}$ & $\begin{array}{l}-0.002^{*} \\
(0.001)\end{array}$ & $\begin{array}{l}-0.000 \\
(0.000)\end{array}$ \\
\hline $\begin{array}{l}\text { Migration back- } \\
\text { ground }\end{array}$ & $\begin{array}{l}-0.059^{\#} \\
(0.035)\end{array}$ & $\begin{array}{l}-0.077 \\
(0.086)\end{array}$ & $\begin{array}{l}-0.415 \\
(0.383)\end{array}$ & $\begin{array}{l}0.242^{\#} \\
(0.131)\end{array}$ & $\begin{array}{l}0.058 \\
(0.146)\end{array}$ & $\begin{array}{l}0.189 \\
(0.208)\end{array}$ & $\begin{array}{l}0.036 \\
(0.024)\end{array}$ \\
\hline \multicolumn{8}{|c|}{ Education (R: elementary) (CASMIN) } \\
\hline Secondary & $\begin{array}{l}0.045 \\
(0.034)\end{array}$ & $\begin{array}{l}0.224^{* *} \\
(0.078)\end{array}$ & $\begin{array}{l}1.038^{* *} \\
(0.380)\end{array}$ & $\begin{array}{l}0.209 \\
(0.131)\end{array}$ & $\begin{array}{l}-0.153 \\
(0.138)\end{array}$ & $\begin{array}{l}-0.205 \\
(0.216)\end{array}$ & $\begin{array}{l}0.019 \\
(0.021)\end{array}$ \\
\hline Maturity & $\begin{array}{l}0.043 \\
(0.048)\end{array}$ & $\begin{array}{l}0.204^{\#} \\
(0.114)\end{array}$ & $\begin{array}{l}0.737 \\
(0.523)\end{array}$ & $\begin{array}{l}0.265 \\
(0.180)\end{array}$ & $\begin{array}{l}0.055 \\
(0.212)\end{array}$ & $\begin{array}{l}0.267 \\
(0.275)\end{array}$ & $\begin{array}{l}0.018 \\
(0.033)\end{array}$ \\
\hline Tertiary & $\begin{array}{l}0.151^{* *} \\
(0.048)\end{array}$ & $\begin{array}{l}0.387^{* * *} \\
(0.106)\end{array}$ & $\begin{array}{l}1.759^{* *} \\
(0.538)\end{array}$ & $\begin{array}{l}0.380^{*} \\
(0.151)\end{array}$ & $\begin{array}{l}0.331 \\
(0.213)\end{array}$ & $\begin{array}{l}-0.155 \\
(0.266)\end{array}$ & $\begin{array}{l}0.112^{* * *} \\
(0.034)\end{array}$ \\
\hline Married & $\begin{array}{l}-0.039 \\
(0.038)\end{array}$ & $\begin{array}{l}-0.054 \\
(0.095)\end{array}$ & $\begin{array}{l}-0.253 \\
(0.410)\end{array}$ & $\begin{array}{l}-0.092 \\
(0.154)\end{array}$ & $\begin{array}{l}-0.110 \\
(0.166)\end{array}$ & $\begin{array}{l}0.088 \\
(0.227)\end{array}$ & $\begin{array}{l}0.004 \\
(0.025)\end{array}$ \\
\hline Household size & $\begin{array}{l}-0.011 \\
(0.015)\end{array}$ & $\begin{array}{l}-0.060 \\
(0.040)\end{array}$ & $\begin{array}{l}-0.242 \\
(0.167)\end{array}$ & $\begin{array}{l}-0.136^{*} \\
(0.059)\end{array}$ & $\begin{array}{l}-0.105 \\
(0.082)\end{array}$ & $\begin{array}{l}-0.044 \\
(0.083)\end{array}$ & $\begin{array}{l}-0.011 \\
(0.011)\end{array}$ \\
\hline Household income & $\begin{array}{l}0.006^{*} \\
(0.003)\end{array}$ & $\begin{array}{l}0.017^{*} \\
(0.008)\end{array}$ & $\begin{array}{l}0.073^{*} \\
(0.033)\end{array}$ & $\begin{array}{l}0.026^{*} \\
(0.011)\end{array}$ & $\begin{array}{l}0.016 \\
(0.015)\end{array}$ & $\begin{array}{l}-0.008 \\
(0.018)\end{array}$ & $\begin{array}{l}0.006^{* *} \\
(0.002)\end{array}$ \\
\hline West Germany & $\begin{array}{l}-0.041 \\
(0.030)\end{array}$ & $\begin{array}{l}-0.154^{*} \\
(0.072)\end{array}$ & $\begin{array}{l}-0.601^{\#} \\
(0.333)\end{array}$ & $\begin{array}{l}-0.297^{*} \\
(0.120)\end{array}$ & $\begin{array}{l}-0.068 \\
(0.130)\end{array}$ & $\begin{array}{l}0.160 \\
(0.206)\end{array}$ & $\begin{array}{l}-0.004 \\
(0.019)\end{array}$ \\
\hline Constant & $\begin{array}{l}-0.256 \\
(0.206)\end{array}$ & $\begin{array}{l}-1.808^{* * *} \\
(0.533)\end{array}$ & $\begin{array}{l}-4.379^{*} \\
(2.208)\end{array}$ & $\begin{array}{l}-2.280^{* *} \\
(0.836)\end{array}$ & $\begin{array}{l}-0.318 \\
(0.896)\end{array}$ & $\begin{array}{l}-3.347^{*} \\
(1.445)\end{array}$ & $\begin{array}{l}0.286^{*} \\
(0.142)\end{array}$ \\
\hline Observations & 1278 & 1278 & 1278 & 1278 & 1278 & 1278 & 1573 \\
\hline
\end{tabular}

Weighted to account for panel attrition from wave 7 to 8 ; multiple imputation by chained equations to account for missing values data (40 imputations, burn in period of 40 iterations). Goodness-of-fit tests not available after multiple imputation in STATA (see STATA multiple imputation reference manual release 13, p. 79)

Outcome variables Models 1 to 5: job search activity (yes/no); number of job search methods; job search intensity; number of hours spent searching; number of applications; outcome variables Models 6 and 7: number job interviews; employed (yes/no)

Robust standard errors in parentheses; ${ }^{* * *} \mathrm{p}<0.001,{ }^{* *} \mathrm{p}<0.01,{ }^{*} \mathrm{p}<0.05,{ }^{*} \mathrm{p}<0.10$

Estimation methods: ${ }^{a}$ linear regression, ${ }^{b}$ negative binomial count data regression 
Table 8 Effects of unemployment stigma after controlling for the initial situation during the job search (as available)

\begin{tabular}{|c|c|c|c|c|c|}
\hline & $\begin{array}{l}\text { H1: Subjective } \\
\text { well-being } \\
\text { Model } 1^{\mathrm{a}}\end{array}$ & $\begin{array}{l}\text { H2: Health } \\
\text { Model 2 }^{\mathrm{a}}\end{array}$ & 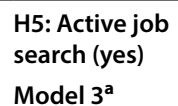 & $\begin{array}{l}\text { H5: Search intensity } \\
\text { Model } 4^{\mathrm{a}}\end{array}$ & $\begin{array}{l}\text { H6: Number } \\
\text { of interviews } \\
\text { Model } 5^{\mathrm{a}}\end{array}$ \\
\hline Stigma consciousness (0-10) & $\begin{array}{l}-0.196^{* * *} \\
(0.029)\end{array}$ & $\begin{array}{l}-0.114^{* *} \\
(0.037)\end{array}$ & $\begin{array}{l}0.014^{*} \\
(0.007)\end{array}$ & $\begin{array}{l}0.044^{*} \\
(0.019)\end{array}$ & $\begin{array}{l}0.095^{*} \\
(0.048)\end{array}$ \\
\hline Initial subjective well-being & $\begin{array}{l}0.462^{* * * *} \\
(0.026)\end{array}$ & & & & \\
\hline Initial health satisfaction & & $\begin{array}{l}0.439^{* * *} \\
(0.032)\end{array}$ & & & \\
\hline Initial active job search & & & $\begin{array}{l}0.443^{* * *} \\
(0.029)\end{array}$ & & \\
\hline Initial number job search methods & & & & $\begin{array}{l}0.265^{* * *} \\
(0.018)\end{array}$ & \\
\hline Initial number of interviews & & & & & $\begin{array}{l}0.525^{* * *} \\
(0.124)\end{array}$ \\
\hline Sample (R: population sample) & $\begin{array}{l}-0.141 \\
(0.212)\end{array}$ & $\begin{array}{l}0.008 \\
(0.276)\end{array}$ & $\begin{array}{l}0.051 \\
(0.054)\end{array}$ & $\begin{array}{l}0.170 \\
(0.136)\end{array}$ & $\begin{array}{l}1.053^{*} \\
(0.427)\end{array}$ \\
\hline \multicolumn{6}{|l|}{ Employment status at wave 6 (R: employed) } \\
\hline Unemployed & $\begin{array}{l}0.034 \\
(0.159)\end{array}$ & $\begin{array}{l}0.175 \\
(0.237)\end{array}$ & $\begin{array}{l}-0.236^{* * *} \\
(0.043)\end{array}$ & $\begin{array}{l}-0.453^{* * *} \\
(0.105)\end{array}$ & $\begin{array}{l}-0.289 \\
(0.240)\end{array}$ \\
\hline Out of the labour force & $\begin{array}{l}0.439 \\
(0.542)\end{array}$ & $\begin{array}{l}-0.040 \\
(0.688)\end{array}$ & $\begin{array}{l}-0.270^{\#} \\
(0.139)\end{array}$ & $\begin{array}{l}-0.540 \\
(0.346)\end{array}$ & $\begin{array}{l}-0.580 \\
(0.622)\end{array}$ \\
\hline Male & $\begin{array}{l}-0.181^{\#} \\
(0.102)\end{array}$ & $\begin{array}{l}-0.063 \\
(0.132)\end{array}$ & $\begin{array}{l}0.015 \\
(0.026)\end{array}$ & $\begin{array}{l}0.038 \\
(0.068)\end{array}$ & $\begin{array}{l}0.569^{* *} \\
(0.192)\end{array}$ \\
\hline Unemployment duration (years) & $\begin{array}{l}0.003 \\
(0.013)\end{array}$ & $\begin{array}{l}-0.031^{\#} \\
(0.016)\end{array}$ & $\begin{array}{l}-0.006^{*} \\
(0.003)\end{array}$ & $\begin{array}{l}-0.023^{*} \\
(0.009)\end{array}$ & $\begin{array}{l}-0.140^{* * *} \\
(0.031)\end{array}$ \\
\hline Number of previous unemployment episodes & $\begin{array}{l}-0.088 \\
(0.059)\end{array}$ & $\begin{array}{l}0.041 \\
(0.084)\end{array}$ & $\begin{array}{l}-0.003 \\
(0.017)\end{array}$ & $\begin{array}{l}0.016 \\
(0.042)\end{array}$ & $\begin{array}{l}0.080 \\
(0.132)\end{array}$ \\
\hline Age & $\begin{array}{l}-0.052 \\
(0.035)\end{array}$ & $\begin{array}{l}-0.167^{* * *} \\
(0.047)\end{array}$ & $\begin{array}{l}0.029^{* *} \\
(0.009)\end{array}$ & $\begin{array}{l}0.113^{* * *} \\
(0.025)\end{array}$ & $\begin{array}{l}0.149^{*} \\
(0.063)\end{array}$ \\
\hline $\mathrm{Age}^{2}$ & $\begin{array}{l}0.001 \\
(0.000)\end{array}$ & $\begin{array}{l}0.002^{* *} \\
(0.001)\end{array}$ & $\begin{array}{l}-0.000^{* * *} \\
(0.000)\end{array}$ & $\begin{array}{l}-0.001^{* * *} \\
(0.000)\end{array}$ & $\begin{array}{l}-0.002^{* *} \\
(0.001)\end{array}$ \\
\hline Migration background & $\begin{array}{l}0.069 \\
(0.124)\end{array}$ & $\begin{array}{l}0.174 \\
(0.159)\end{array}$ & $\begin{array}{l}-0.074^{*} \\
(0.031)\end{array}$ & $\begin{array}{l}-0.126 \\
(0.086)\end{array}$ & $\begin{array}{l}0.125 \\
(0.192)\end{array}$ \\
\hline \multicolumn{6}{|l|}{ Education (R: elementary) (CASMIN) } \\
\hline Secondary & $\begin{array}{l}-0.017 \\
(0.114)\end{array}$ & $\begin{array}{l}-0.195 \\
(0.156)\end{array}$ & $\begin{array}{l}0.013 \\
(0.032)\end{array}$ & $\begin{array}{l}0.185^{*} \\
(0.080)\end{array}$ & $\begin{array}{l}-0.369^{\#} \\
(0.216)\end{array}$ \\
\hline Maturity & $\begin{array}{l}-0.056 \\
(0.172)\end{array}$ & $\begin{array}{l}0.425^{*} \\
(0.211)\end{array}$ & $\begin{array}{l}-0.021 \\
(0.044)\end{array}$ & $\begin{array}{l}-0.031 \\
(0.106)\end{array}$ & $\begin{array}{l}-0.009 \\
(0.258)\end{array}$ \\
\hline Tertiary & $\begin{array}{l}-0.161 \\
(0.185)\end{array}$ & $\begin{array}{l}0.268 \\
(0.219)\end{array}$ & $\begin{array}{l}0.063 \\
(0.043)\end{array}$ & $\begin{array}{l}0.084 \\
(0.106)\end{array}$ & $\begin{array}{l}-0.300 \\
(0.271)\end{array}$ \\
\hline Married & $\begin{array}{l}-0.022 \\
(0.133)\end{array}$ & $\begin{array}{l}0.011 \\
(0.167)\end{array}$ & $\begin{array}{l}-0.032 \\
(0.035)\end{array}$ & $\begin{array}{l}-0.124 \\
(0.095)\end{array}$ & $\begin{array}{l}0.030 \\
(0.223)\end{array}$ \\
\hline Household size & $\begin{array}{l}0.048 \\
(0.053)\end{array}$ & $\begin{array}{l}0.045 \\
(0.078)\end{array}$ & $\begin{array}{l}0.010 \\
(0.015)\end{array}$ & $\begin{array}{l}0.002 \\
(0.039)\end{array}$ & $\begin{array}{l}0.054 \\
(0.087)\end{array}$ \\
\hline Household income & $\begin{array}{l}-0.001 \\
(0.010)\end{array}$ & $\begin{array}{l}0.012 \\
(0.014)\end{array}$ & $\begin{array}{l}0.004 \\
(0.003)\end{array}$ & $\begin{array}{l}0.014^{\#} \\
(0.008)\end{array}$ & $\begin{array}{l}-0.011 \\
(0.017)\end{array}$ \\
\hline West Germany & $\begin{array}{l}0.004 \\
(0.108)\end{array}$ & $\begin{array}{l}-0.383^{* *} \\
(0.143)\end{array}$ & $\begin{array}{l}-0.025 \\
(0.028)\end{array}$ & $\begin{array}{l}-0.087 \\
(0.073)\end{array}$ & $\begin{array}{l}0.123 \\
(0.211)\end{array}$ \\
\hline Constant & $\begin{array}{l}5.536^{* * * *} \\
(0.778)\end{array}$ & $\begin{array}{l}7.285^{* * *} \\
(1.146)\end{array}$ & $\begin{array}{l}-0.093 \\
(0.194)\end{array}$ & $\begin{array}{l}-2.039^{* * *} \\
(0.538)\end{array}$ & $\begin{array}{l}-5.035^{* * *} \\
(1.436)\end{array}$ \\
\hline Observations & 1278 & 1278 & 1278 & 1278 & 1278 \\
\hline
\end{tabular}

Weighted to account for panel attrition from wave 7 to 8 ; multiple imputation by chained equations to account for missing values data (40 imputations, burn in period of 40 iterations). Goodness-of-fit tests not available after multiple imputation in STATA (see STATA multiple imputation reference manual release 13, p. 79)

Outcome variables Models 1 to 5: Life satisfaction (11-pt. scale); health satisfaction (11-pt. scale); job search activity (yes/no); number of job search methods; number of job interviews

Robust standard errors in parentheses; ${ }^{* * *} \mathrm{p}<0.001,{ }^{* *} \mathrm{p}<0.01,{ }^{*} \mathrm{p}<0.05,{ }^{*} \mathrm{p}<0.10$

Estimation methods: alinear regression 
Table 9 Effects of unemployment stigma on subjective well-being (H1), health (H2), re-employment expectations (H3) and value placed on employment ( $\mathrm{H} 4$ ) after controlling for pressure by an employment office

\begin{tabular}{|c|c|c|c|c|}
\hline & $\begin{array}{l}\text { H1: Subjective well- } \\
\text { being }\end{array}$ & H2: Health & H3: Expectations & H4: Value \\
\hline & & & & \\
\hline Stigma consciousness $(0-10)$ & $\begin{array}{l}-0.310^{* * *} \\
(0.034)\end{array}$ & $\begin{array}{l}-0.204^{* * *} \\
(0.041)\end{array}$ & $\begin{array}{l}-0.043^{* *} \\
(0.014)\end{array}$ & $\begin{array}{l}0.129^{* * *} \\
(0.017)\end{array}$ \\
\hline Sample (R: population sample) & - & - & - & - \\
\hline \multicolumn{5}{|l|}{ Integration agreement signed (R: yes) } \\
\hline No agreement signed & $\begin{array}{l}0.257 \\
(0.195)\end{array}$ & $\begin{array}{l}0.058 \\
(0.234)\end{array}$ & $\begin{array}{l}0.044 \\
(0.082)\end{array}$ & $\begin{array}{l}0.151 \\
(0.111)\end{array}$ \\
\hline Does not apply & $\begin{array}{l}0.176 \\
(0.338)\end{array}$ & $\begin{array}{l}0.166 \\
(0.378)\end{array}$ & $\begin{array}{l}0.281^{\#} \\
(0.145)\end{array}$ & $\begin{array}{l}0.199 \\
(0.166)\end{array}$ \\
\hline Experiences with job center (negative to positive) & $\begin{array}{l}-0.241^{* *} \\
(0.075)\end{array}$ & $\begin{array}{l}-0.027 \\
(0.090)\end{array}$ & $\begin{array}{l}-0.116^{* * *} \\
(0.029)\end{array}$ & $\begin{array}{l}-0.128^{* * *} \\
(0.035)\end{array}$ \\
\hline \multicolumn{5}{|l|}{ Employment status at wave 6 (R: employed) } \\
\hline Unemployed & $\begin{array}{l}-0.336^{\#} \\
(0.196)\end{array}$ & $\begin{array}{l}-0.192 \\
(0.259)\end{array}$ & $\begin{array}{l}-0.196^{*} \\
(0.089)\end{array}$ & $\begin{array}{l}-0.203^{*} \\
(0.099)\end{array}$ \\
\hline Out of the labour force & $\begin{array}{l}-0.236 \\
(0.578)\end{array}$ & $\begin{array}{l}-0.387 \\
(0.759)\end{array}$ & $\begin{array}{l}-0.019 \\
(0.315)\end{array}$ & $\begin{array}{l}0.201 \\
(0.357)\end{array}$ \\
\hline Male & $\begin{array}{l}-0.358^{* *} \\
(0.118)\end{array}$ & $\begin{array}{l}-0.088 \\
(0.149)\end{array}$ & $\begin{array}{l}0.068 \\
(0.051)\end{array}$ & $\begin{array}{l}-0.158^{*} \\
(0.062)\end{array}$ \\
\hline Unemployment duration (years) & $\begin{array}{l}0.007 \\
(0.015)\end{array}$ & $\begin{array}{l}-0.038^{\#} \\
(0.020)\end{array}$ & $\begin{array}{l}-0.018^{* * *} \\
(0.005)\end{array}$ & $\begin{array}{l}0.015^{*} \\
(0.007)\end{array}$ \\
\hline Number of previous unemployment episodes & $\begin{array}{l}-0.060 \\
(0.075)\end{array}$ & $\begin{array}{l}0.156 \\
(0.098)\end{array}$ & $\begin{array}{l}0.044 \\
(0.034)\end{array}$ & $\begin{array}{l}0.018 \\
(0.034)\end{array}$ \\
\hline Age & $\begin{array}{l}-0.102^{*} \\
(0.040)\end{array}$ & $\begin{array}{l}-0.286^{* * *} \\
(0.050)\end{array}$ & $\begin{array}{l}0.000 \\
(0.017)\end{array}$ & $\begin{array}{l}0.039^{\#} \\
(0.022)\end{array}$ \\
\hline $\mathrm{Age}^{2}$ & $\begin{array}{l}0.001^{*} \\
(0.000)\end{array}$ & $\begin{array}{l}0.003^{* * *} \\
(0.001)\end{array}$ & $\begin{array}{l}-0.000 \\
(0.000)\end{array}$ & $\begin{array}{l}-0.000 \\
(0.000)\end{array}$ \\
\hline Migration background & $\begin{array}{l}0.279^{*} \\
(0.139)\end{array}$ & $\begin{array}{l}0.388^{*} \\
(0.181)\end{array}$ & $\begin{array}{l}0.085 \\
(0.065)\end{array}$ & $\begin{array}{l}-0.002 \\
(0.075)\end{array}$ \\
\hline \multicolumn{5}{|l|}{ Education (R: elementary) (CASMIN) } \\
\hline Secondary & $\begin{array}{l}-0.112 \\
(0.136)\end{array}$ & $\begin{array}{l}-0.258 \\
(0.176)\end{array}$ & $\begin{array}{l}-0.041 \\
(0.061)\end{array}$ & $\begin{array}{l}-0.114 \\
(0.074)\end{array}$ \\
\hline Maturity & $\begin{array}{l}-0.172 \\
(0.184)\end{array}$ & $\begin{array}{l}0.481^{*} \\
(0.231)\end{array}$ & $\begin{array}{l}0.074 \\
(0.089)\end{array}$ & $\begin{array}{l}-0.350^{* * *} \\
(0.094)\end{array}$ \\
\hline Tertiary & $\begin{array}{l}-0.150 \\
(0.219)\end{array}$ & $\begin{array}{l}0.411 \\
(0.252)\end{array}$ & $\begin{array}{l}0.076 \\
(0.092)\end{array}$ & $\begin{array}{l}-0.338^{* *} \\
(0.104)\end{array}$ \\
\hline Married & $\begin{array}{l}0.106 \\
(0.155)\end{array}$ & $\begin{array}{l}-0.232 \\
(0.188)\end{array}$ & $\begin{array}{l}-0.055 \\
(0.071)\end{array}$ & $\begin{array}{l}0.148^{\#} \\
(0.087)\end{array}$ \\
\hline Household size & $\begin{array}{l}0.077 \\
(0.063)\end{array}$ & $\begin{array}{l}0.109 \\
(0.087)\end{array}$ & $\begin{array}{l}-0.025 \\
(0.034)\end{array}$ & $\begin{array}{l}-0.010 \\
(0.041)\end{array}$ \\
\hline Household income & $\begin{array}{l}0.010 \\
(0.015)\end{array}$ & $\begin{array}{l}0.012 \\
(0.019)\end{array}$ & $\begin{array}{l}0.001 \\
(0.007)\end{array}$ & $\begin{array}{l}0.011 \\
(0.008)\end{array}$ \\
\hline West Germany & $\begin{array}{l}-0.041 \\
(0.127)\end{array}$ & $\begin{array}{l}-0.463^{* *} \\
(0.159)\end{array}$ & $\begin{array}{l}0.042 \\
(0.056)\end{array}$ & $\begin{array}{l}-0.099 \\
(0.067)\end{array}$ \\
\hline Constant & $\begin{array}{l}9.936^{* * *} \\
(0.857)\end{array}$ & $\begin{array}{l}13.078^{* * *} \\
(1.113)\end{array}$ & $\begin{array}{l}2.874^{* * *} \\
(0.380)\end{array}$ & $\begin{array}{l}-1.584^{* *} \\
(0.483)\end{array}$ \\
\hline Observations & 1198 & 1198 & 1198 & 1198 \\
\hline
\end{tabular}

Weighted to account for panel attrition from wave 7 to 8 ; multiple imputation by chained equations to account for missing values data (40 imputations, burn in period of 30 iterations). Goodness-of-fit tests not available after multiple imputation in STATA (see STATA multiple imputation reference manual release 13, p. 79)

Outcome variables Models 1 to 4: Life satisfaction (11-pt. scale); health satisfaction (11-pt. scale); self-assessed re-employment chances (4-pt. scale) factor score nonmonetary motivation to work (continuous)

Robust standard errors in parentheses; ${ }^{* * *} \mathrm{p}<0.001,{ }^{* *} \mathrm{p}<0.01,{ }^{*} \mathrm{p}<0.05,{ }^{*} \mathrm{p}<0.10$; Sample contains only unemployed in sgb-II context (welfare benefit)

Estimation methods: ${ }^{a}$ linear regression 
Table 10 Effects of unemployment stigma on job search effort (H5) and success (H6) after controlling for pressure by an employment office

\begin{tabular}{|c|c|c|c|c|c|c|c|}
\hline & \multicolumn{5}{|c|}{ H5a/b: Job search effort } & \multicolumn{2}{|c|}{ H6a/b: Job search success } \\
\hline & $\begin{array}{l}\text { Active job search } \\
\text { (yes) }\end{array}$ & $\begin{array}{l}\text { Number } \\
\text { of methods }\end{array}$ & Search intensity & Hours searched & $\begin{array}{l}\text { Number } \\
\text { of applications }\end{array}$ & $\begin{array}{l}\text { Number } \\
\text { of interviews }\end{array}$ & Job (yes) \\
\hline & Model $1^{\mathrm{a}}$ & Model $2^{b}$ & Model $3^{c}$ & Model $4^{b}$ & Model $5^{b}$ & Model $6^{b}$ & Model $7^{\mathrm{a}}$ \\
\hline $\begin{array}{l}\text { Stigma conscious- } \\
\text { ness (0-10) }\end{array}$ & $\begin{array}{l}0.030^{* * *} \\
(0.008)\end{array}$ & $\begin{array}{l}0.073^{* * *} \\
(0.019)\end{array}$ & $\begin{array}{l}0.323^{* * *} \\
(0.084)\end{array}$ & $\begin{array}{l}0.089^{*} \\
(0.036)\end{array}$ & $\begin{array}{l}0.073^{*} \\
(0.035)\end{array}$ & $\begin{array}{l}0.070 \\
(0.050)\end{array}$ & $\begin{array}{l}-0.005 \\
(0.005)\end{array}$ \\
\hline $\begin{array}{l}\text { Sample (R: popula- } \\
\text { tion sample) }\end{array}$ & - & - & - & - & - & - & - \\
\hline \multicolumn{8}{|c|}{ Integration agreement signed (R: yes) } \\
\hline $\begin{array}{l}\text { No agreement } \\
\text { signed }\end{array}$ & $\begin{array}{l}-0.044 \\
(0.049)\end{array}$ & $\begin{array}{l}-0.222^{\#} \\
(0.129)\end{array}$ & $\begin{array}{l}-0.886^{\#} \\
(0.513)\end{array}$ & $\begin{array}{l}-0.373^{*} \\
(0.175)\end{array}$ & $\begin{array}{l}-0.508^{*} \\
(0.204)\end{array}$ & $\begin{array}{l}-0.377 \\
(0.358)\end{array}$ & $\begin{array}{l}0.011 \\
(0.029)\end{array}$ \\
\hline Does not apply & $\begin{array}{l}-0.022 \\
(0.082)\end{array}$ & $\begin{array}{l}-0.066 \\
(0.167)\end{array}$ & $\begin{array}{l}-0.413 \\
(0.839)\end{array}$ & $\begin{array}{l}-0.235 \\
(0.232)\end{array}$ & $\begin{array}{l}-0.266 \\
(0.304)\end{array}$ & $\begin{array}{l}-0.700 \\
(0.456)\end{array}$ & $\begin{array}{l}0.083 \\
(0.059)\end{array}$ \\
\hline $\begin{array}{l}\text { Experiences with job } \\
\text { center (negative to } \\
\text { positive) }\end{array}$ & $\begin{array}{l}-0.031^{\#} \\
(0.017)\end{array}$ & $\begin{array}{l}-0.053 \\
(0.040)\end{array}$ & $\begin{array}{l}-0.333^{\#} \\
(0.187)\end{array}$ & $\begin{array}{l}-0.101 \\
(0.067)\end{array}$ & $\begin{array}{l}-0.046 \\
(0.070)\end{array}$ & $\begin{array}{l}-0.192^{\#} \\
(0.116)\end{array}$ & $\begin{array}{l}0.010 \\
(0.011)\end{array}$ \\
\hline \multicolumn{8}{|c|}{ Employment status at wave 6 (R: employed) } \\
\hline Unemployed & $\begin{array}{l}-0.055 \\
(0.044)\end{array}$ & $\begin{array}{l}-0.021 \\
(0.096)\end{array}$ & $\begin{array}{l}0.027 \\
(0.481)\end{array}$ & $\begin{array}{l}0.129 \\
(0.151)\end{array}$ & $\begin{array}{l}-0.079 \\
(0.185)\end{array}$ & $\begin{array}{l}-0.256 \\
(0.280)\end{array}$ & $\begin{array}{l}-0.147^{* * *} \\
(0.033)\end{array}$ \\
\hline $\begin{array}{l}\text { Out of the labour } \\
\text { force }\end{array}$ & $\begin{array}{l}0.067 \\
(0.132)\end{array}$ & $\begin{array}{l}-0.048 \\
(0.306)\end{array}$ & $\begin{array}{l}0.941 \\
(1.736)\end{array}$ & $\begin{array}{l}0.181 \\
(0.450)\end{array}$ & $\begin{array}{l}-0.908^{\#} \\
(0.507)\end{array}$ & $\begin{array}{l}-0.546 \\
(0.606)\end{array}$ & $\begin{array}{l}-0.084 \\
(0.111)\end{array}$ \\
\hline Male & $\begin{array}{l}0.055^{\#} \\
(0.029)\end{array}$ & $\begin{array}{l}0.118^{\#} \\
(0.070)\end{array}$ & $\begin{array}{l}0.634^{*} \\
(0.319)\end{array}$ & $\begin{array}{l}0.308^{* *} \\
(0.110)\end{array}$ & $\begin{array}{l}0.199 \\
(0.128)\end{array}$ & $\begin{array}{l}0.555^{* *} \\
(0.190)\end{array}$ & $\begin{array}{l}0.007 \\
(0.018)\end{array}$ \\
\hline $\begin{array}{l}\text { Unemployment dura- } \\
\text { tion (years) }\end{array}$ & $\begin{array}{l}-0.011^{* * *} \\
(0.003)\end{array}$ & $\begin{array}{l}-0.033^{* * *} \\
(0.010)\end{array}$ & $\begin{array}{l}-0.117^{* * *} \\
(0.033)\end{array}$ & $\begin{array}{l}-0.061^{* * *} \\
(0.014)\end{array}$ & $\begin{array}{l}-0.061^{* * *} \\
(0.016)\end{array}$ & $\begin{array}{l}-0.171^{* * *} \\
(0.031)\end{array}$ & $\begin{array}{l}-0.005^{* *} \\
(0.002)\end{array}$ \\
\hline $\begin{array}{l}\text { Number of previous } \\
\text { unemployment } \\
\text { episodes }\end{array}$ & $\begin{array}{l}0.022 \\
(0.019)\end{array}$ & $\begin{array}{l}0.059 \\
(0.043)\end{array}$ & $\begin{array}{l}0.299 \\
(0.222)\end{array}$ & $\begin{array}{l}0.041 \\
(0.066)\end{array}$ & $\begin{array}{l}0.070 \\
(0.071)\end{array}$ & $\begin{array}{l}0.046 \\
(0.133)\end{array}$ & $\begin{array}{l}0.033^{*} \\
(0.014)\end{array}$ \\
\hline Age & $\begin{array}{l}0.038^{* * *} \\
(0.010)\end{array}$ & $\begin{array}{l}0.120^{* * *} \\
(0.026)\end{array}$ & $\begin{array}{l}0.443^{* * *} \\
(0.105)\end{array}$ & $\begin{array}{l}0.161^{* * *} \\
(0.039)\end{array}$ & $\begin{array}{l}0.092^{*} \\
(0.042)\end{array}$ & $\begin{array}{l}0.104^{\#} \\
(0.063)\end{array}$ & $\begin{array}{l}0.004 \\
(0.006)\end{array}$ \\
\hline $\mathrm{Age}^{2}$ & $\begin{array}{l}-0.000^{* * *} \\
(0.000)\end{array}$ & $\begin{array}{l}-0.002^{* * *} \\
(0.000)\end{array}$ & $\begin{array}{l}-0.006^{* * *} \\
(0.001)\end{array}$ & $\begin{array}{l}-0.002^{* * *} \\
(0.000)\end{array}$ & $\begin{array}{l}-0.001^{* *} \\
(0.000)\end{array}$ & $\begin{array}{l}-0.002^{*} \\
(0.001)\end{array}$ & $\begin{array}{l}-0.000 \\
(0.000)\end{array}$ \\
\hline $\begin{array}{l}\text { Migration back- } \\
\text { ground }\end{array}$ & $\begin{array}{l}-0.070^{\#} \\
(0.035)\end{array}$ & $\begin{array}{l}-0.088 \\
(0.088)\end{array}$ & $\begin{array}{l}-0.463 \\
(0.392)\end{array}$ & $\begin{array}{l}0.190 \\
(0.130)\end{array}$ & $\begin{array}{l}0.015 \\
(0.155)\end{array}$ & $\begin{array}{l}0.106 \\
(0.203)\end{array}$ & $\begin{array}{l}0.036 \\
(0.024)\end{array}$ \\
\hline \multicolumn{8}{|c|}{ Education (R: elementary) (CASMIN) } \\
\hline Secondary & $\begin{array}{l}0.021 \\
(0.035)\end{array}$ & $\begin{array}{l}0.173^{*} \\
(0.082)\end{array}$ & $\begin{array}{l}0.876^{*} \\
(0.395)\end{array}$ & $\begin{array}{l}0.127 \\
(0.137)\end{array}$ & $\begin{array}{l}-0.143 \\
(0.144)\end{array}$ & $\begin{array}{l}-0.317 \\
(0.228)\end{array}$ & $\begin{array}{l}0.015 \\
(0.022)\end{array}$ \\
\hline Maturity & $\begin{array}{l}0.015 \\
(0.049)\end{array}$ & $\begin{array}{l}0.144 \\
(0.118)\end{array}$ & $\begin{array}{l}0.457 \\
(0.538)\end{array}$ & $\begin{array}{l}0.184 \\
(0.192)\end{array}$ & $\begin{array}{l}-0.021 \\
(0.222)\end{array}$ & $\begin{array}{l}0.048 \\
(0.274)\end{array}$ & $\begin{array}{l}0.010 \\
(0.033)\end{array}$ \\
\hline Tertiary & $\begin{array}{l}0.124^{*} \\
(0.050)\end{array}$ & $\begin{array}{l}0.300^{* *} \\
(0.109)\end{array}$ & $\begin{array}{l}1.408^{*} \\
(0.558)\end{array}$ & $\begin{array}{l}0.239 \\
(0.160)\end{array}$ & $\begin{array}{l}0.183 \\
(0.218)\end{array}$ & $\begin{array}{l}-0.247 \\
(0.277)\end{array}$ & $\begin{array}{l}0.095^{* *} \\
(0.035)\end{array}$ \\
\hline Married & $\begin{array}{l}-0.055 \\
(0.039)\end{array}$ & $\begin{array}{l}-0.114 \\
(0.100)\end{array}$ & $\begin{array}{l}-0.449 \\
(0.427)\end{array}$ & $\begin{array}{l}-0.143 \\
(0.167)\end{array}$ & $\begin{array}{l}-0.142 \\
(0.179)\end{array}$ & $\begin{array}{l}0.038 \\
(0.238)\end{array}$ & $\begin{array}{l}-0.014 \\
(0.025)\end{array}$ \\
\hline Household size & $\begin{array}{l}-0.010 \\
(0.017)\end{array}$ & $\begin{array}{l}-0.052 \\
(0.045)\end{array}$ & $\begin{array}{l}-0.224 \\
(0.185)\end{array}$ & $\begin{array}{l}-0.132^{*} \\
(0.063)\end{array}$ & $\begin{array}{l}-0.090 \\
(0.090)\end{array}$ & $\begin{array}{l}-0.009 \\
(0.096)\end{array}$ & $\begin{array}{l}-0.012 \\
(0.012)\end{array}$ \\
\hline Household income & $\begin{array}{l}0.007^{\#} \\
(0.004)\end{array}$ & $\begin{array}{l}0.022^{*} \\
(0.009)\end{array}$ & $\begin{array}{l}0.092^{*} \\
(0.038)\end{array}$ & $\begin{array}{l}0.038^{* *} \\
(0.013)\end{array}$ & $\begin{array}{l}0.028 \\
(0.018)\end{array}$ & $\begin{array}{l}-0.004 \\
(0.020)\end{array}$ & $\begin{array}{l}0.008^{* *} \\
(0.003)\end{array}$ \\
\hline West Germany & $\begin{array}{l}-0.061^{\#} \\
(0.032)\end{array}$ & $\begin{array}{l}-0.201^{* *} \\
(0.076)\end{array}$ & $\begin{array}{l}-0.751^{*} \\
(0.345)\end{array}$ & $\begin{array}{l}-0.353^{* *} \\
(0.126)\end{array}$ & $\begin{array}{l}-0.114 \\
(0.138)\end{array}$ & $\begin{array}{l}0.024 \\
(0.210)\end{array}$ & $\begin{array}{l}0.002 \\
(0.020)\end{array}$ \\
\hline Constant & $\begin{array}{l}-0.214 \\
(0.211)\end{array}$ & $\begin{array}{l}-1.917^{* * *} \\
(0.544)\end{array}$ & $\begin{array}{l}-4.765^{*} \\
(2.289)\end{array}$ & $\begin{array}{l}-2.209^{* *} \\
(0.837)\end{array}$ & $\begin{array}{l}-0.103 \\
(0.905)\end{array}$ & $\begin{array}{l}-2.366^{\#} \\
(1.393)\end{array}$ & $\begin{array}{l}0.207 \\
(0.144)\end{array}$ \\
\hline Observations & 1198 & 1198 & 1198 & 1198 & 1198 & 1198 & 1471 \\
\hline
\end{tabular}

Weighted to account for panel attrition from wave 7 to 8 ; multiple imputation by chained equations to account for missing values data ( 40 imputations, burn in period of 40 iterations). Goodness-of-fit tests not available after multiple imputation in STATA (see STATA multiple imputation reference manual release 13, p. 79)

Outcome variables Models 1 to 5 : job search activity (yes/no); number of job search methods; job search intensity; number of hours spent searching; number of applications; Outcome Variables Models 6 and 7: number job interviews; employed (yes/no)

Robust standard errors in parentheses; ${ }^{* * *} \mathrm{p}<0.001,{ }^{* *} \mathrm{p}<0.01,{ }^{*} \mathrm{p}<0.05,{ }^{*} \mathrm{p}<0.10$; Sample contains only unemployed in sgb-II context (welfare benefit)

Estimation methods: ${ }^{a}$ linear regression, ${ }^{b}$ negative binomial count data regression 
Table 11 Effects of unemployment stigma on subjective well-being (H1), health (H2), re-employment expectations (H3) and value placed on employment (H4) after controlling for personality traits

\begin{tabular}{|c|c|c|c|c|}
\hline & $\begin{array}{l}\text { H1: Subjective well-being } \\
\text { Model } 1^{\text {a }}\end{array}$ & $\begin{array}{l}\text { H2: Health } \\
\text { Model } 2^{\mathrm{a}}\end{array}$ & $\begin{array}{l}\text { H3: Expectations } \\
\text { Model } 3^{\text {a }}\end{array}$ & $\begin{array}{l}\text { H4: Value } \\
\text { Model } 4^{\mathrm{a}}\end{array}$ \\
\hline Stigma consciousness (0-10) & $\begin{array}{l}-0.235^{* * *} \\
(0.035)\end{array}$ & $\begin{array}{l}-0.127^{* *} \\
(0.041)\end{array}$ & $\begin{array}{l}-0.034^{*} \\
(0.014)\end{array}$ & $\begin{array}{l}0.143^{* * *} \\
(0.017)\end{array}$ \\
\hline Sample (R: population sample) & $\begin{array}{l}-0.011 \\
(0.020)\end{array}$ & $\begin{array}{l}-0.027 \\
(0.028)\end{array}$ & $\begin{array}{l}0.005 \\
(0.009)\end{array}$ & $\begin{array}{l}-0.007 \\
(0.011)\end{array}$ \\
\hline Extraversion & $\begin{array}{l}0.010 \\
(0.024)\end{array}$ & $\begin{array}{l}-0.014 \\
(0.029)\end{array}$ & $\begin{array}{l}0.001 \\
(0.010)\end{array}$ & $\begin{array}{l}0.023^{\#} \\
(0.013)\end{array}$ \\
\hline Agreeableness & $\begin{array}{l}0.025 \\
(0.036)\end{array}$ & $\begin{array}{l}0.047 \\
(0.044)\end{array}$ & $\begin{array}{l}-0.013 \\
(0.016)\end{array}$ & $\begin{array}{l}0.032 \\
(0.020)\end{array}$ \\
\hline Conscientiousness & $\begin{array}{l}0.033 \\
(0.032)\end{array}$ & $\begin{array}{l}-0.022 \\
(0.045)\end{array}$ & $\begin{array}{l}0.005 \\
(0.014)\end{array}$ & $\begin{array}{l}-0.022 \\
(0.018)\end{array}$ \\
\hline Neuroticism & $\begin{array}{l}-0.033^{\#} \\
(0.020)\end{array}$ & $\begin{array}{l}0.009 \\
(0.026)\end{array}$ & $\begin{array}{l}0.009 \\
(0.009)\end{array}$ & $\begin{array}{l}0.008 \\
(0.009)\end{array}$ \\
\hline Openness to experience & $\begin{array}{l}-0.193^{* * *} \\
(0.025)\end{array}$ & $\begin{array}{l}-0.164^{* * *} \\
(0.030)\end{array}$ & $\begin{array}{l}-0.029^{* *} \\
(0.011)\end{array}$ & $\begin{array}{l}-0.043^{* * *} \\
(0.012)\end{array}$ \\
\hline Self-efficacy & $\begin{array}{l}-0.152 \\
(0.236)\end{array}$ & $\begin{array}{l}0.038 \\
(0.303)\end{array}$ & $\begin{array}{l}0.063 \\
(0.101)\end{array}$ & $\begin{array}{l}0.032 \\
(0.140)\end{array}$ \\
\hline \multicolumn{5}{|l|}{ Employment status at wave 6 (R: employed) } \\
\hline Unemployed & $\begin{array}{l}-0.226 \\
(0.171)\end{array}$ & $\begin{array}{l}-0.049 \\
(0.252)\end{array}$ & $\begin{array}{l}-0.183^{*} \\
(0.083)\end{array}$ & $\begin{array}{l}-0.183^{\#} \\
(0.095)\end{array}$ \\
\hline Out of the labour force & $\begin{array}{l}-0.059 \\
(0.556)\end{array}$ & $\begin{array}{l}-0.112 \\
(0.721)\end{array}$ & $\begin{array}{l}-0.015 \\
(0.318)\end{array}$ & $\begin{array}{l}0.332 \\
(0.366)\end{array}$ \\
\hline Male & $\begin{array}{l}-0.383^{* * *} \\
(0.113)\end{array}$ & $\begin{array}{l}-0.094 \\
(0.144)\end{array}$ & $\begin{array}{l}0.064 \\
(0.049)\end{array}$ & $\begin{array}{l}-0.180^{* *} \\
(0.060)\end{array}$ \\
\hline Unemployment duration (years) & $\begin{array}{l}-0.002 \\
(0.014)\end{array}$ & $\begin{array}{l}-0.041^{*} \\
(0.018)\end{array}$ & $\begin{array}{l}-0.018^{* * *} \\
(0.005)\end{array}$ & $\begin{array}{l}0.013^{\#} \\
(0.007)\end{array}$ \\
\hline Number of previous unemployment episodes & $\begin{array}{l}-0.070 \\
(0.069)\end{array}$ & $\begin{array}{l}0.133 \\
(0.093)\end{array}$ & $\begin{array}{l}0.044 \\
(0.032)\end{array}$ & $\begin{array}{l}0.027 \\
(0.033)\end{array}$ \\
\hline Age & $\begin{array}{l}-0.090^{*} \\
(0.038)\end{array}$ & $\begin{array}{l}-0.283^{* * *} \\
(0.047)\end{array}$ & $\begin{array}{l}0.008 \\
(0.017)\end{array}$ & $\begin{array}{l}0.033 \\
(0.021)\end{array}$ \\
\hline $\mathrm{Age}^{2}$ & $\begin{array}{l}0.001^{*} \\
(0.000)\end{array}$ & $\begin{array}{l}0.003^{* * *} \\
(0.001)\end{array}$ & $\begin{array}{l}-0.000^{*} \\
(0.000)\end{array}$ & $\begin{array}{l}-0.000 \\
(0.000)\end{array}$ \\
\hline Migration background & $\begin{array}{l}0.227^{\#} \\
(0.133)\end{array}$ & $\begin{array}{l}0.302^{\#} \\
(0.174)\end{array}$ & $\begin{array}{l}0.070 \\
(0.063)\end{array}$ & $\begin{array}{l}-0.025 \\
(0.073)\end{array}$ \\
\hline \multicolumn{5}{|l|}{ Education (R: elementary) (CASMIN) } \\
\hline Secondary & $\begin{array}{l}-0.025 \\
(0.128)\end{array}$ & $\begin{array}{l}-0.164 \\
(0.168)\end{array}$ & $\begin{array}{l}-0.016 \\
(0.061)\end{array}$ & $\begin{array}{l}-0.138^{\#} \\
(0.073)\end{array}$ \\
\hline Maturity & $\begin{array}{l}0.022 \\
(0.177)\end{array}$ & $\begin{array}{l}0.532^{*} \\
(0.227)\end{array}$ & $\begin{array}{l}0.066 \\
(0.087)\end{array}$ & $\begin{array}{l}-0.350^{* * *} \\
(0.092)\end{array}$ \\
\hline Tertiary & $\begin{array}{l}-0.068 \\
(0.213)\end{array}$ & $\begin{array}{l}0.492^{*} \\
(0.250)\end{array}$ & $\begin{array}{l}0.103 \\
(0.091)\end{array}$ & $\begin{array}{l}-0.365^{* * *} \\
(0.103)\end{array}$ \\
\hline Married & $\begin{array}{l}0.202 \\
(0.148)\end{array}$ & $\begin{array}{l}-0.066 \\
(0.184)\end{array}$ & $\begin{array}{l}-0.024 \\
(0.068)\end{array}$ & $\begin{array}{l}0.216^{*} \\
(0.085)\end{array}$ \\
\hline Household size & $\begin{array}{l}0.050 \\
(0.055)\end{array}$ & $\begin{array}{l}0.080 \\
(0.080)\end{array}$ & $\begin{array}{l}-0.034 \\
(0.030)\end{array}$ & $\begin{array}{l}-0.008 \\
(0.037)\end{array}$ \\
\hline Household income & $\begin{array}{l}0.002 \\
(0.011)\end{array}$ & $\begin{array}{l}0.012 \\
(0.015)\end{array}$ & $\begin{array}{l}0.001 \\
(0.005)\end{array}$ & $\begin{array}{l}0.008 \\
(0.006)\end{array}$ \\
\hline West Germany & $\begin{array}{l}0.120 \\
(0.121)\end{array}$ & $\begin{array}{l}-0.380^{*} \\
(0.153)\end{array}$ & $\begin{array}{l}0.088 \\
(0.055)\end{array}$ & $\begin{array}{l}-0.037 \\
(0.066)\end{array}$ \\
\hline Constant & $\begin{array}{l}11.508^{* * *} \\
(1.189)\end{array}$ & $\begin{array}{l}14.235^{* * *} \\
(1.592)\end{array}$ & $\begin{array}{l}2.833^{* * *} \\
(0.534)\end{array}$ & $\begin{array}{l}-1.588^{*} \\
(0.690)\end{array}$ \\
\hline Observations & 1278 & 1278 & 1278 & 1278 \\
\hline
\end{tabular}

Weighted to account for panel attrition from wave 7 to 8 ; multiple imputation by chained equations to account for missing values data (40 imputations, burn in period of 30 iterations). Goodness-of-fit tests not available after multiple imputation in STATA (see STATA multiple imputation reference manual release 13, p. 79)

Outcome variables Models 1 to 4: Life satisfaction (11-pt. scale); health satisfaction (11-pt. scale); self-assessed re-employment chances (4-pt. scale) factor score nonmonetary motivation to work (continuous)

Robust standard errors in parentheses; ${ }^{* * *} p<0.001,{ }^{* *} p<0.01,{ }^{*} p<0.05,{ }^{*} p<0.10$

Estimation methods: alinear regression 
Table 12 Effects of unemployment stigma on job search effort (H5) and success (H6) after controlling for personality traits

\begin{tabular}{|c|c|c|c|c|c|c|c|}
\hline & \multicolumn{5}{|c|}{ H5a/b: Job search effort } & \multicolumn{2}{|c|}{ H6a/b: Job search success } \\
\hline & $\begin{array}{l}\text { Active job search } \\
\text { (yes) }\end{array}$ & $\begin{array}{l}\text { Number } \\
\text { of methods }\end{array}$ & Search intensity & Hours searched & $\begin{array}{l}\text { Number } \\
\text { of applications }\end{array}$ & $\begin{array}{l}\text { Number } \\
\text { of interviews }\end{array}$ & Job (yes) \\
\hline & Model $1^{\mathrm{a}}$ & Model $2^{b}$ & Model $3^{a}$ & Model $4^{\mathrm{b}}$ & Model $5^{b}$ & Model $6^{b}$ & Model $7^{\mathrm{a}}$ \\
\hline $\begin{array}{l}\text { Stigma conscious- } \\
\text { ness }(0-10)\end{array}$ & $\begin{array}{l}0.030^{* * *} \\
(0.008)\end{array}$ & $\begin{array}{l}0.072^{* * *} \\
(0.020)\end{array}$ & $\begin{array}{l}0.285^{* * *} \\
(0.085)\end{array}$ & $\begin{array}{l}0.089^{* *} \\
(0.034)\end{array}$ & $\begin{array}{l}0.082^{*} \\
(0.037)\end{array}$ & $\begin{array}{l}0.097^{\#} \\
(0.052)\end{array}$ & $\begin{array}{l}-0.002 \\
(0.005)\end{array}$ \\
\hline $\begin{array}{l}\text { Sample (R: popula- } \\
\text { tion sample) }\end{array}$ & $\begin{array}{l}0.001 \\
(0.005)\end{array}$ & $\begin{array}{l}0.002 \\
(0.012)\end{array}$ & $\begin{array}{l}-0.036 \\
(0.053)\end{array}$ & $\begin{array}{l}0.025 \\
(0.019)\end{array}$ & $\begin{array}{l}0.040^{\#} \\
(0.023)\end{array}$ & $\begin{array}{l}0.055^{\#} \\
(0.033)\end{array}$ & $\begin{array}{l}-0.003 \\
(0.003)\end{array}$ \\
\hline Extraversion & $\begin{array}{l}-0.001 \\
(0.006)\end{array}$ & $\begin{array}{l}0.001 \\
(0.013)\end{array}$ & $\begin{array}{l}-0.011 \\
(0.062)\end{array}$ & $\begin{array}{l}-0.020 \\
(0.021)\end{array}$ & $\begin{array}{l}0.010 \\
(0.025)\end{array}$ & $\begin{array}{l}-0.006 \\
(0.037)\end{array}$ & $\begin{array}{l}0.001 \\
(0.004)\end{array}$ \\
\hline Agreeableness & $\begin{array}{l}0.018^{*} \\
(0.008)\end{array}$ & $\begin{array}{l}0.059^{* *} \\
(0.021)\end{array}$ & $\begin{array}{l}0.237^{*} \\
(0.094)\end{array}$ & $\begin{array}{l}0.102^{* *} \\
(0.032)\end{array}$ & $\begin{array}{l}0.100^{*} \\
(0.041)\end{array}$ & $\begin{array}{l}0.124^{*} \\
(0.057)\end{array}$ & $\begin{array}{l}0.016^{*} \\
(0.007)\end{array}$ \\
\hline Conscientiousness & $\begin{array}{l}-0.020^{*} \\
(0.008)\end{array}$ & $\begin{array}{l}-0.046^{*} \\
(0.021)\end{array}$ & $\begin{array}{l}-0.276^{* *} \\
(0.095)\end{array}$ & $\begin{array}{l}-0.022 \\
(0.031)\end{array}$ & $\begin{array}{l}-0.047 \\
(0.036)\end{array}$ & $\begin{array}{l}-0.086 \\
(0.054)\end{array}$ & $\begin{array}{l}-0.013^{*} \\
(0.005)\end{array}$ \\
\hline Neuroticism & $\begin{array}{l}-0.006 \\
(0.005)\end{array}$ & $\begin{array}{l}-0.017 \\
(0.013)\end{array}$ & $\begin{array}{l}-0.075 \\
(0.056)\end{array}$ & $\begin{array}{l}-0.009 \\
(0.018)\end{array}$ & $\begin{array}{l}0.000 \\
(0.023)\end{array}$ & $\begin{array}{l}0.018 \\
(0.033)\end{array}$ & $\begin{array}{l}-0.005 \\
(0.003)\end{array}$ \\
\hline $\begin{array}{l}\text { Openness to } \\
\text { experience }\end{array}$ & $\begin{array}{l}-0.006 \\
(0.005)\end{array}$ & $\begin{array}{l}-0.007 \\
(0.014)\end{array}$ & $\begin{array}{l}0.003 \\
(0.058)\end{array}$ & $\begin{array}{l}0.010 \\
(0.022)\end{array}$ & $\begin{array}{l}-0.017 \\
(0.026)\end{array}$ & $\begin{array}{l}-0.004 \\
(0.036)\end{array}$ & $\begin{array}{l}-0.003 \\
(0.003)\end{array}$ \\
\hline Self-efficacy & $\begin{array}{l}0.039 \\
(0.060)\end{array}$ & $\begin{array}{l}0.055 \\
(0.152)\end{array}$ & $\begin{array}{l}0.469 \\
(0.616)\end{array}$ & $\begin{array}{l}0.328 \\
(0.214)\end{array}$ & $\begin{array}{l}0.098 \\
(0.273)\end{array}$ & $\begin{array}{l}1.103^{*} \\
(0.430)\end{array}$ & $\begin{array}{l}0.013 \\
(0.034)\end{array}$ \\
\hline \multicolumn{8}{|c|}{ Employment status at wave 6 (R: employed) } \\
\hline Unemployed & $\begin{array}{l}-0.036 \\
(0.045)\end{array}$ & $\begin{array}{l}-0.000 \\
(0.097)\end{array}$ & $\begin{array}{l}0.014 \\
(0.471)\end{array}$ & $\begin{array}{l}0.188 \\
(0.154)\end{array}$ & $\begin{array}{l}0.000 \\
(0.180)\end{array}$ & $\begin{array}{l}-0.279 \\
(0.265)\end{array}$ & $\begin{array}{l}-0.144^{* * *} \\
(0.033)\end{array}$ \\
\hline $\begin{array}{l}\text { Out of the labour } \\
\text { force }\end{array}$ & $\begin{array}{l}0.098 \\
(0.146)\end{array}$ & $\begin{array}{l}0.072 \\
(0.320)\end{array}$ & $\begin{array}{l}1.259 \\
(1.785)\end{array}$ & $\begin{array}{l}0.426 \\
(0.482)\end{array}$ & $\begin{array}{l}-0.645 \\
(0.541)\end{array}$ & $\begin{array}{l}-0.155 \\
(0.613)\end{array}$ & $\begin{array}{l}0.029 \\
(0.115)\end{array}$ \\
\hline Male & $\begin{array}{l}0.044 \\
(0.028)\end{array}$ & $\begin{array}{l}0.093 \\
(0.069)\end{array}$ & $\begin{array}{l}0.487 \\
(0.312)\end{array}$ & $\begin{array}{l}0.276^{* *} \\
(0.107)\end{array}$ & $\begin{array}{l}0.207 \\
(0.128)\end{array}$ & $\begin{array}{l}0.562^{* *} \\
(0.188)\end{array}$ & $\begin{array}{l}0.001 \\
(0.018)\end{array}$ \\
\hline $\begin{array}{l}\text { Unemployment } \\
\text { duration (years) }\end{array}$ & $\begin{array}{l}-0.010^{* * *} \\
(0.003)\end{array}$ & $\begin{array}{l}-0.035^{* * *} \\
(0.009)\end{array}$ & $\begin{array}{l}-0.117^{* * *} \\
(0.032)\end{array}$ & $\begin{array}{l}-0.064^{* * *} \\
(0.014)\end{array}$ & $\begin{array}{l}-0.060^{* * *} \\
(0.015)\end{array}$ & $\begin{array}{l}-0.154^{* * *} \\
(0.030)\end{array}$ & $\begin{array}{l}-0.006^{* * *} \\
(0.002)\end{array}$ \\
\hline $\begin{array}{l}\text { Number of previous } \\
\text { unemployment } \\
\text { episodes }\end{array}$ & $\begin{array}{l}0.019 \\
(0.018)\end{array}$ & $\begin{array}{l}0.058 \\
(0.041)\end{array}$ & $\begin{array}{l}0.315 \\
(0.209)\end{array}$ & $\begin{array}{l}-0.004 \\
(0.058)\end{array}$ & $\begin{array}{l}0.035 \\
(0.067)\end{array}$ & $\begin{array}{l}-0.013 \\
(0.108)\end{array}$ & $\begin{array}{l}0.042^{* *} \\
(0.013)\end{array}$ \\
\hline Age & $\begin{array}{l}0.039^{* * *} \\
(0.009)\end{array}$ & $\begin{array}{l}0.121^{* * *} \\
(0.025)\end{array}$ & $\begin{array}{l}0.428^{* * *} \\
(0.101)\end{array}$ & $\begin{array}{l}0.171^{* * *} \\
(0.037)\end{array}$ & $\begin{array}{l}0.117^{* *} \\
(0.042)\end{array}$ & $\begin{array}{l}0.121^{*} \\
(0.058)\end{array}$ & $\begin{array}{l}0.003 \\
(0.006)\end{array}$ \\
\hline $\mathrm{Age}^{2}$ & $\begin{array}{l}-0.001^{* * *} \\
(0.000)\end{array}$ & $\begin{array}{l}-0.002^{* * *} \\
(0.000)\end{array}$ & $\begin{array}{l}-0.005^{* * *} \\
(0.001)\end{array}$ & $\begin{array}{l}-0.002^{* * *} \\
(0.000)\end{array}$ & $\begin{array}{l}-0.002^{* * *} \\
(0.000)\end{array}$ & $\begin{array}{l}-0.002^{* *} \\
(0.001)\end{array}$ & $\begin{array}{l}-0.000 \\
(0.000)\end{array}$ \\
\hline $\begin{array}{l}\text { Migration back- } \\
\text { ground }\end{array}$ & $\begin{array}{l}-0.068^{*} \\
(0.035)\end{array}$ & $\begin{array}{l}-0.112 \\
(0.088)\end{array}$ & $\begin{array}{l}-0.509 \\
(0.379)\end{array}$ & $\begin{array}{l}0.167 \\
(0.131)\end{array}$ & $\begin{array}{l}-0.063 \\
(0.153)\end{array}$ & $\begin{array}{l}0.068 \\
(0.196)\end{array}$ & $\begin{array}{l}0.035 \\
(0.023)\end{array}$ \\
\hline \multicolumn{8}{|c|}{ Education (R: elementary) (CASMIN) } \\
\hline Secondary & $\begin{array}{l}0.035 \\
(0.034)\end{array}$ & $\begin{array}{l}0.211^{* *} \\
(0.080)\end{array}$ & $\begin{array}{l}0.938^{*} \\
(0.381)\end{array}$ & $\begin{array}{l}0.192 \\
(0.131)\end{array}$ & $\begin{array}{l}-0.130 \\
(0.144)\end{array}$ & $\begin{array}{l}-0.230 \\
(0.221)\end{array}$ & $\begin{array}{l}0.010 \\
(0.021)\end{array}$ \\
\hline Maturity & $\begin{array}{l}0.039 \\
(0.049)\end{array}$ & $\begin{array}{l}0.195^{\#} \\
(0.118)\end{array}$ & $\begin{array}{l}0.694 \\
(0.539)\end{array}$ & $\begin{array}{l}0.208 \\
(0.185)\end{array}$ & $\begin{array}{l}-0.006 \\
(0.216)\end{array}$ & $\begin{array}{l}0.098 \\
(0.269)\end{array}$ & $\begin{array}{l}0.006 \\
(0.032)\end{array}$ \\
\hline Tertiary & $\begin{array}{l}0.133^{* *} \\
(0.050)\end{array}$ & $\begin{array}{l}0.339^{* *} \\
(0.113)\end{array}$ & $\begin{array}{l}1.572^{* *} \\
(0.557)\end{array}$ & $\begin{array}{l}0.304^{\#} \\
(0.162)\end{array}$ & $\begin{array}{l}0.218 \\
(0.222)\end{array}$ & $\begin{array}{l}-0.375 \\
(0.276)\end{array}$ & $\begin{array}{l}0.097^{* *} \\
(0.035)\end{array}$ \\
\hline Married & $\begin{array}{l}-0.050 \\
(0.038)\end{array}$ & $\begin{array}{l}-0.109 \\
(0.097)\end{array}$ & $\begin{array}{l}-0.436 \\
(0.408)\end{array}$ & $\begin{array}{l}-0.154 \\
(0.154)\end{array}$ & $\begin{array}{l}-0.125 \\
(0.176)\end{array}$ & $\begin{array}{l}0.069 \\
(0.234)\end{array}$ & $\begin{array}{l}-0.007 \\
(0.025)\end{array}$ \\
\hline Household size & $\begin{array}{l}-0.008 \\
(0.015)\end{array}$ & $\begin{array}{l}-0.038 \\
(0.042)\end{array}$ & $\begin{array}{l}-0.210 \\
(0.169)\end{array}$ & $\begin{array}{l}-0.087 \\
(0.058)\end{array}$ & $\begin{array}{l}-0.053 \\
(0.080)\end{array}$ & $\begin{array}{l}0.083 \\
(0.089)\end{array}$ & $\begin{array}{l}-0.007 \\
(0.011)\end{array}$ \\
\hline Household income & $\begin{array}{l}0.005^{\#} \\
(0.003)\end{array}$ & $\begin{array}{l}0.014^{\#} \\
(0.008)\end{array}$ & $\begin{array}{l}0.067^{*} \\
(0.032)\end{array}$ & $\begin{array}{l}0.025^{*} \\
(0.011)\end{array}$ & $\begin{array}{l}0.011 \\
(0.014)\end{array}$ & $\begin{array}{l}-0.021 \\
(0.018)\end{array}$ & $\begin{array}{l}0.005^{*} \\
(0.002)\end{array}$ \\
\hline West Germany & $\begin{array}{l}-0.056^{\#} \\
(0.031)\end{array}$ & $\begin{array}{l}-0.198^{* *} \\
(0.074)\end{array}$ & $\begin{array}{l}-0.786^{*} \\
(0.333)\end{array}$ & $\begin{array}{l}-0.408^{* * *} \\
(0.121)\end{array}$ & $\begin{array}{l}-0.206 \\
(0.131)\end{array}$ & $\begin{array}{l}0.015 \\
(0.210)\end{array}$ & $\begin{array}{l}-0.011 \\
(0.019)\end{array}$ \\
\hline Constant & $\begin{array}{l}-0.140 \\
(0.289)\end{array}$ & $\begin{array}{l}-1.971^{* *} \\
(0.724)\end{array}$ & $\begin{array}{l}-3.032 \\
(3.165)\end{array}$ & $\begin{array}{l}-4.041^{* * *} \\
(1.098)\end{array}$ & $\begin{array}{l}-2.092 \\
(1.336)\end{array}$ & $\begin{array}{l}-5.872^{* * *} \\
(1.756)\end{array}$ & $\begin{array}{l}0.260 \\
(0.203)\end{array}$ \\
\hline Observations & 1278 & 1278 & 1278 & 1278 & 1278 & 1278 & 1573 \\
\hline
\end{tabular}


Table 12 (continued)

Weighted to account for panel attrition from wave 7 to 8 ; multiple imputation by chained equations to account for missing values data (40 imputations, burn in period of 40 iterations). Goodness-of-fit tests not available after multiple imputation in STATA (see STATA multiple imputation reference manual release 13, p. 79) Outcome variables Models 1 to 5 : job search activity (yes/no); number of job search methods; job search intensity; number of hours spent searching; number of applications; Outcome variables Models 6 and 7: number of job interviews; employed (yes/no)

Robust standard errors in parentheses; ${ }^{* * *} p<0.001,{ }^{* *} p<0.01,{ }^{*} p<0.05,{ }^{*} p<0.10$

Estimation methods: ${ }^{a}$ linear regression, ${ }^{b}$ negative binomial count data regression

Table 13 Effects of unemployment stigma on subjective well-being (H1), health (H2), re-employment expectations (H3) and value placed on employment (H4) without imputation

\begin{tabular}{|c|c|c|c|c|}
\hline & $\begin{array}{l}\text { H1: Subjective well-being } \\
\text { Model } 1^{\text {a }}\end{array}$ & $\begin{array}{l}\text { H2: Health } \\
\text { Model } 2^{\mathrm{a}}\end{array}$ & $\begin{array}{l}\text { H3: Expectations } \\
\text { Model } 3^{a}\end{array}$ & $\begin{array}{l}\text { H4: Value } \\
\text { Model } 4^{\mathrm{a}}\end{array}$ \\
\hline Stigma consciousness $(0-10)$ & $\begin{array}{l}-0.323^{* * *} \\
(0.035)\end{array}$ & $\begin{array}{l}-0.193^{* * *} \\
(0.044)\end{array}$ & $\begin{array}{l}-0.042^{* *} \\
(0.015)\end{array}$ & $\begin{array}{l}0.122^{* * *} \\
(0.018)\end{array}$ \\
\hline Sample (R: population sample) & $\begin{array}{l}-0.242 \\
(0.243)\end{array}$ & $\begin{array}{l}0.044 \\
(0.313)\end{array}$ & $\begin{array}{l}0.027 \\
(0.106)\end{array}$ & $\begin{array}{l}-0.021 \\
(0.142)\end{array}$ \\
\hline \multicolumn{5}{|l|}{ Employment status at wave 6 (R: employed) } \\
\hline Unemployed & $\begin{array}{l}-0.244 \\
(0.178)\end{array}$ & $\begin{array}{l}-0.036 \\
(0.247)\end{array}$ & $\begin{array}{l}-0.177^{*} \\
(0.083)\end{array}$ & $\begin{array}{l}-0.207^{*} \\
(0.094)\end{array}$ \\
\hline Out of the labour force & $\begin{array}{l}0.152 \\
(0.485)\end{array}$ & $\begin{array}{l}-0.118 \\
(0.746)\end{array}$ & $\begin{array}{l}0.109 \\
(0.323)\end{array}$ & $\begin{array}{l}0.301 \\
(0.356)\end{array}$ \\
\hline Male & $\begin{array}{l}-0.294^{*} \\
(0.124)\end{array}$ & $\begin{array}{l}-0.047 \\
(0.160)\end{array}$ & $\begin{array}{l}0.019 \\
(0.054)\end{array}$ & $\begin{array}{l}-0.196^{* *} \\
(0.065)\end{array}$ \\
\hline Unemployment duration (years) & $\begin{array}{l}0.009 \\
(0.015)\end{array}$ & $\begin{array}{l}-0.039^{\#} \\
(0.021)\end{array}$ & $\begin{array}{l}-0.018^{* *} \\
(0.005)\end{array}$ & $\begin{array}{l}0.012 \\
(0.007)\end{array}$ \\
\hline Number of previous unemployment episodes & $\begin{array}{l}-0.051 \\
(0.071)\end{array}$ & $\begin{array}{l}0.125 \\
(0.106)\end{array}$ & $\begin{array}{l}0.047 \\
(0.036)\end{array}$ & $\begin{array}{l}0.033 \\
(0.038)\end{array}$ \\
\hline Age & $\begin{array}{l}-0.057 \\
(0.043)\end{array}$ & $\begin{array}{l}-0.220^{* * *} \\
(0.057)\end{array}$ & $\begin{array}{l}0.017 \\
(0.019)\end{array}$ & $\begin{array}{l}0.017 \\
(0.023)\end{array}$ \\
\hline $\mathrm{Age}^{2}$ & $\begin{array}{l}0.001 \\
(0.000)\end{array}$ & $\begin{array}{l}0.002^{* * *} \\
(0.001)\end{array}$ & $\begin{array}{l}-0.000^{*} \\
(0.000)\end{array}$ & $\begin{array}{l}-0.000 \\
(0.000)\end{array}$ \\
\hline Migration background & $\begin{array}{l}0.287^{\#} \\
(0.150)\end{array}$ & $\begin{array}{l}0.411^{*} \\
(0.191)\end{array}$ & $\begin{array}{l}0.039 \\
(0.068)\end{array}$ & $\begin{array}{l}-0.044 \\
(0.080)\end{array}$ \\
\hline \multicolumn{5}{|l|}{ Education (R: Elementary) (CASMIN) } \\
\hline Secondary & $\begin{array}{l}-0.068 \\
(0.140)\end{array}$ & $\begin{array}{l}-0.074 \\
(0.187)\end{array}$ & $\begin{array}{l}-0.094 \\
(0.063)\end{array}$ & $\begin{array}{l}-0.189^{*} \\
(0.081)\end{array}$ \\
\hline Maturity & $\begin{array}{l}-0.217 \\
(0.209)\end{array}$ & $\begin{array}{l}0.649^{*} \\
(0.265)\end{array}$ & $\begin{array}{l}0.110 \\
(0.097)\end{array}$ & $\begin{array}{l}-0.349^{* * *} \\
(0.100)\end{array}$ \\
\hline Tertiary & $\begin{array}{l}-0.280 \\
(0.221)\end{array}$ & $\begin{array}{l}0.621^{*} \\
(0.264)\end{array}$ & $\begin{array}{l}0.071 \\
(0.094)\end{array}$ & $\begin{array}{l}-0.311^{* *} \\
(0.109)\end{array}$ \\
\hline Married & $\begin{array}{l}0.028 \\
(0.164)\end{array}$ & $\begin{array}{l}-0.239 \\
(0.208)\end{array}$ & $\begin{array}{l}-0.067 \\
(0.076)\end{array}$ & $\begin{array}{l}0.255^{* *} \\
(0.090)\end{array}$ \\
\hline Household size & $\begin{array}{l}0.076 \\
(0.062)\end{array}$ & $\begin{array}{l}0.137 \\
(0.093)\end{array}$ & $\begin{array}{l}-0.022 \\
(0.033)\end{array}$ & $\begin{array}{l}-0.026 \\
(0.040)\end{array}$ \\
\hline Household income & $\begin{array}{l}0.015 \\
(0.012)\end{array}$ & $\begin{array}{l}0.026^{\#} \\
(0.015)\end{array}$ & $\begin{array}{l}0.007 \\
(0.006)\end{array}$ & $\begin{array}{l}0.009 \\
(0.007)\end{array}$ \\
\hline West Germany & $\begin{array}{l}-0.041 \\
(0.134)\end{array}$ & $\begin{array}{l}-0.410^{*} \\
(0.173)\end{array}$ & $\begin{array}{l}0.047 \\
(0.060)\end{array}$ & $\begin{array}{l}-0.081 \\
(0.073)\end{array}$ \\
\hline Constant & $\begin{array}{l}8.841^{* * *} \\
(0.904)\end{array}$ & $\begin{array}{l}11.005^{* * *} \\
(1.273)\end{array}$ & $\begin{array}{l}2.392^{* * * *} \\
(0.431)\end{array}$ & $\begin{array}{l}-0.971^{\#} \\
(0.516)\end{array}$ \\
\hline Observations & 1025 & 1026 & 1016 & 1021 \\
\hline R-squared & 0.111 & 0.099 & 0.167 & 0.103 \\
\hline
\end{tabular}

Weighted to account for panel attrition from wave 7 to 8; Outcome variables Models 1 to 5 : job search activity (yes/no); number of job search methods; job search intensity; number of hours spent searching; number of applications; Outcome variables Models 1 to 4: Life satisfaction (11-pt. scale); health satisfaction (11-pt. scale); self-assessed re-employment chances (4-pt. scale) factor score non-monetary motivation to work (continuous)

Estimation methods: ${ }^{\text {linear regression }}$ 
Table 14 Effects of unemployment stigma on job search effort (H5) and success (H6) without imputation

\begin{tabular}{|c|c|c|c|c|c|c|c|}
\hline & \multicolumn{5}{|c|}{ H5a/b: Job search effort } & \multicolumn{2}{|c|}{ H6a/b: Job search success } \\
\hline & Model $1^{\mathrm{a}}$ & Model $2^{\mathbf{b}}$ & Model $3^{a}$ & Model $4^{b}$ & Model $5^{b}$ & Model $6^{\mathrm{b}}$ & Model $7^{a}$ \\
\hline & $\begin{array}{l}\text { Active job search } \\
\text { (yes) }\end{array}$ & $\begin{array}{l}\text { Number } \\
\text { methods }\end{array}$ & Search intensity & Hours searched & $\begin{array}{l}\text { Number } \\
\text { applications }\end{array}$ & $\begin{array}{l}\text { Number } \\
\text { interviews }\end{array}$ & Job (yes) \\
\hline $\begin{array}{l}\text { Stigma conscious- } \\
\text { ness }(0-10)\end{array}$ & $\begin{array}{l}0.022^{* *} \\
(0.008)\end{array}$ & $\begin{array}{l}0.054^{* *} \\
(0.021)\end{array}$ & $\begin{array}{l}0.248^{* *} \\
(0.090)\end{array}$ & $\begin{array}{l}0.062^{\#} \\
(0.037)\end{array}$ & $\begin{array}{l}0.051 \\
(0.038)\end{array}$ & $\begin{array}{l}0.060 \\
(0.056)\end{array}$ & $\begin{array}{l}-0.007 \\
(0.005)\end{array}$ \\
\hline $\begin{array}{l}\text { Sample (R: popu- } \\
\text { lation sample) }\end{array}$ & $\begin{array}{l}0.053 \\
(0.061)\end{array}$ & $\begin{array}{l}0.162 \\
(0.153)\end{array}$ & $\begin{array}{l}0.965 \\
(0.600)\end{array}$ & $\begin{array}{l}0.436^{\#} \\
(0.226)\end{array}$ & $\begin{array}{l}0.269 \\
(0.288)\end{array}$ & $\begin{array}{l}1.193^{*} \\
(0.529)\end{array}$ & $\begin{array}{l}0.003 \\
(0.035)\end{array}$ \\
\hline \multicolumn{8}{|c|}{ Employment status at wave 6 (R: employed) } \\
\hline Unemployed & $\begin{array}{l}-0.042 \\
(0.044)\end{array}$ & $\begin{array}{l}0.010 \\
(0.094)\end{array}$ & $\begin{array}{l}-0.027 \\
(0.475)\end{array}$ & $\begin{array}{l}0.211 \\
(0.141)\end{array}$ & $\begin{array}{l}0.026 \\
(0.185)\end{array}$ & $\begin{array}{l}-0.081 \\
(0.245)\end{array}$ & $\begin{array}{l}-0.151^{* * *} \\
(0.032)\end{array}$ \\
\hline $\begin{array}{l}\text { Out of the labour } \\
\text { force }\end{array}$ & $\begin{array}{l}0.061 \\
(0.137)\end{array}$ & $\begin{array}{l}-0.144 \\
(0.303)\end{array}$ & $\begin{array}{l}0.376 \\
(1.823)\end{array}$ & $\begin{array}{l}0.248 \\
(0.442)\end{array}$ & $\begin{array}{l}-1.011^{*} \\
(0.400)\end{array}$ & $\begin{array}{l}-0.341 \\
(0.558)\end{array}$ & $\begin{array}{l}0.024 \\
(0.123)\end{array}$ \\
\hline Male & $\begin{array}{l}0.018 \\
(0.031)\end{array}$ & $\begin{array}{l}0.034 \\
(0.074)\end{array}$ & $\begin{array}{l}0.344 \\
(0.347)\end{array}$ & $\begin{array}{l}0.176 \\
(0.115)\end{array}$ & $\begin{array}{l}0.092 \\
(0.144)\end{array}$ & $\begin{array}{l}0.550^{* *} \\
(0.212)\end{array}$ & $\begin{array}{l}0.018 \\
(0.019)\end{array}$ \\
\hline $\begin{array}{l}\text { Unemployment } \\
\text { duration (years) }\end{array}$ & $\begin{array}{l}-0.013^{* * *} \\
(0.003)\end{array}$ & $\begin{array}{l}-0.044^{* * *} \\
(0.010)\end{array}$ & $\begin{array}{l}-0.151^{* * *} \\
(0.034)\end{array}$ & $\begin{array}{l}-0.066^{* * *} \\
(0.015)\end{array}$ & $\begin{array}{l}-0.080^{* * *} \\
(0.017)\end{array}$ & $\begin{array}{l}-0.159^{* * *} \\
(0.037)\end{array}$ & $\begin{array}{l}-0.004^{*} \\
(0.002)\end{array}$ \\
\hline $\begin{array}{l}\text { Number of previ- } \\
\text { ous unemploy- } \\
\text { ment episodes }\end{array}$ & $\begin{array}{l}0.011 \\
(0.020)\end{array}$ & $\begin{array}{l}0.017 \\
(0.044)\end{array}$ & $\begin{array}{l}0.119 \\
(0.236)\end{array}$ & $\begin{array}{l}0.035 \\
(0.066)\end{array}$ & $\begin{array}{l}-0.001 \\
(0.075)\end{array}$ & $\begin{array}{l}0.014 \\
(0.150)\end{array}$ & $\begin{array}{l}0.054^{* * *} \\
(0.015)\end{array}$ \\
\hline Age & $\begin{array}{l}0.047^{* * *} \\
(0.011)\end{array}$ & $\begin{array}{l}0.124^{* * *} \\
(0.029)\end{array}$ & $\begin{array}{l}0.439^{* * *} \\
(0.121)\end{array}$ & $\begin{array}{l}0.200^{* * *} \\
(0.044)\end{array}$ & $\begin{array}{l}0.130^{* *} \\
(0.050)\end{array}$ & $\begin{array}{l}0.097 \\
(0.073)\end{array}$ & $\begin{array}{l}0.006 \\
(0.007)\end{array}$ \\
\hline $\mathrm{Age}^{2}$ & $\begin{array}{l}-0.001^{* * *} \\
(0.000)\end{array}$ & $\begin{array}{l}-0.002^{* * *} \\
(0.000)\end{array}$ & $\begin{array}{l}-0.006^{* * *} \\
(0.001)\end{array}$ & $\begin{array}{l}-0.002^{* * *} \\
(0.001)\end{array}$ & $\begin{array}{l}-0.002^{* *} \\
(0.001)\end{array}$ & $\begin{array}{l}-0.002^{\#} \\
(0.001)\end{array}$ & $\begin{array}{l}-0.000 \\
(0.000)\end{array}$ \\
\hline $\begin{array}{l}\text { Migration back- } \\
\text { ground }\end{array}$ & $\begin{array}{l}-0.027 \\
(0.038)\end{array}$ & $\begin{array}{l}-0.016 \\
(0.093)\end{array}$ & $\begin{array}{l}-0.143 \\
(0.428)\end{array}$ & $\begin{array}{l}0.231 \\
(0.141)\end{array}$ & $\begin{array}{l}0.066 \\
(0.165)\end{array}$ & $\begin{array}{l}0.155 \\
(0.206)\end{array}$ & $\begin{array}{l}0.021 \\
(0.025)\end{array}$ \\
\hline \multicolumn{8}{|c|}{ Education (R: elementary) (CASMIN) } \\
\hline Secondary & $\begin{array}{l}0.041 \\
(0.038)\end{array}$ & $\begin{array}{l}0.207^{*} \\
(0.086)\end{array}$ & $\begin{array}{l}0.899^{*} \\
(0.415)\end{array}$ & $\begin{array}{l}0.252^{\#} \\
(0.146)\end{array}$ & $\begin{array}{l}-0.093 \\
(0.161)\end{array}$ & $\begin{array}{l}-0.051 \\
(0.251)\end{array}$ & $\begin{array}{l}0.007 \\
(0.023)\end{array}$ \\
\hline Maturity & $\begin{array}{l}0.083 \\
(0.056)\end{array}$ & $\begin{array}{l}0.299^{*} \\
(0.125)\end{array}$ & $\begin{array}{l}1.095^{\#} \\
(0.648)\end{array}$ & $\begin{array}{l}0.190 \\
(0.200)\end{array}$ & $\begin{array}{l}0.208 \\
(0.250)\end{array}$ & $\begin{array}{l}0.329 \\
(0.290)\end{array}$ & $\begin{array}{l}-0.062^{*} \\
(0.031)\end{array}$ \\
\hline Tertiary & $\begin{array}{l}0.139^{* *} \\
(0.053)\end{array}$ & $\begin{array}{l}0.363^{* *} \\
(0.117)\end{array}$ & $\begin{array}{l}1.750^{* *} \\
(0.607)\end{array}$ & $\begin{array}{l}0.358^{*} \\
(0.171)\end{array}$ & $\begin{array}{l}0.285 \\
(0.236)\end{array}$ & $\begin{array}{l}-0.185 \\
(0.297)\end{array}$ & $\begin{array}{l}0.091^{*} \\
(0.036)\end{array}$ \\
\hline Married & $\begin{array}{l}-0.066 \\
(0.042)\end{array}$ & $\begin{array}{l}-0.108 \\
(0.106)\end{array}$ & $\begin{array}{l}-0.412 \\
(0.470)\end{array}$ & $\begin{array}{l}-0.132 \\
(0.164)\end{array}$ & $\begin{array}{l}-0.225 \\
(0.205)\end{array}$ & $\begin{array}{l}0.150 \\
(0.241)\end{array}$ & $\begin{array}{l}-0.004 \\
(0.027)\end{array}$ \\
\hline Household size & $\begin{array}{l}-0.002 \\
(0.017)\end{array}$ & $\begin{array}{l}-0.038 \\
(0.046)\end{array}$ & $\begin{array}{l}-0.190 \\
(0.194)\end{array}$ & $\begin{array}{l}-0.119^{\#} \\
(0.061)\end{array}$ & $\begin{array}{l}-0.010 \\
(0.094)\end{array}$ & $\begin{array}{l}0.030 \\
(0.104)\end{array}$ & $\begin{array}{l}0.004 \\
(0.012)\end{array}$ \\
\hline $\begin{array}{l}\text { Household } \\
\text { income }\end{array}$ & $\begin{array}{l}0.005^{\#} \\
(0.003)\end{array}$ & $\begin{array}{l}0.015^{\#} \\
(0.008)\end{array}$ & $\begin{array}{l}0.068^{\#} \\
(0.036)\end{array}$ & $\begin{array}{l}0.032^{* *} \\
(0.012)\end{array}$ & $\begin{array}{l}0.008 \\
(0.017)\end{array}$ & $\begin{array}{l}-0.017 \\
(0.019)\end{array}$ & $\begin{array}{l}0.003 \\
(0.002)\end{array}$ \\
\hline West Germany & $\begin{array}{l}-0.082^{*} \\
(0.034)\end{array}$ & $\begin{array}{l}-0.249^{* *} \\
(0.081)\end{array}$ & $\begin{array}{l}-1.080^{* *} \\
(0.375)\end{array}$ & $\begin{array}{l}-0.384^{* *} \\
(0.136)\end{array}$ & $\begin{array}{l}-0.134 \\
(0.150)\end{array}$ & $\begin{array}{l}0.115 \\
(0.238)\end{array}$ & $\begin{array}{l}-0.000 \\
(0.021)\end{array}$ \\
\hline Constant & $\begin{array}{l}-0.383 \\
(0.240)\end{array}$ & $\begin{array}{l}-1.879^{* *} \\
(0.626)\end{array}$ & $\begin{array}{l}-4.198 \\
(2.666)\end{array}$ & $\begin{array}{l}-3.340^{* * *} \\
(0.969)\end{array}$ & $\begin{array}{l}-0.939 \\
(1.111)\end{array}$ & $\begin{array}{l}-3.617^{*} \\
(1.660)\end{array}$ & $\begin{array}{l}0.135 \\
(0.162)\end{array}$ \\
\hline Observations & 1027 & 1027 & 1027 & 1020 & 1020 & 1025 & 1260 \\
\hline $\begin{array}{l}\text { (Pseudo) } \\
\text { R-squared }\end{array}$ & 0.097 & & 0.095 & & & & 0.143 \\
\hline
\end{tabular}

Weighted to account for panel attrition from wave 7 to 8; Outcome variables Models 1 to 5: job search activity (yes/no); number of job search methods; job search intensity; number of hours spent searching; number of applications; Outcome variables Models 6 and 7: number of job interviews; employed (yes/no)

Robust standard errors in parentheses; ${ }^{* * *} \mathrm{p}<0.001,{ }^{* *} \mathrm{p}<0.01,{ }^{*} \mathrm{p}<0.05,{ }^{*} \mathrm{p}<0.10$

Estimation methods: ${ }^{a}$ linear regression, ${ }^{b}$ negative binomial count data regression 
Received: 1 February 2018 Accepted: 3 July 2019

Published online: 12 July 2019

\section{References}

Abramson, L.Y., Seligman, M.E., Teasdale, J.D.: Learned helplessness in humans: critique and reformulation. J. Abnorm. Soc. Psychol. 87(1), 49 (1978)

Andrade, C.: The economics of welfare participation and welfare stigma: a review. Public Financ. Manag. 2(2), 294-333 (2002)

Arulampalam, W.: Is unemployment really scarring? Effects of unemployment experiences on wages. Econ. J. 111(475), 585-606 (2001). https://doi. org/10.1111/1468-0297.00664

Arulampalam, W.: State dependence in unemployment incidence: evidence for British men revisited. IZA discussion paper, vol. 630. IZA, Bonn (2002)

Arulampalam, W., Gregg, P., Gregory, M.: Unemployment scarring. Econ. J. 111(475), 577-584 (2001). https://doi.org/10.1111/1468-0297.00663

Ayllón, S.: Understanding poverty persistence in Spain. SERIEs 4(2), 201-233 (2013). https://doi.org/10.1007/s13209-012-0089-4

Baumann, A.E.: Stigmatization, social distance and exclusion because of mental illness. The individual with mental illness as a 'stranger'. Int. Rev. Psychiatry 19(2), 131-135 (2007). https://doi.org/10.1080/0954026070 1278739

Besley, T.J., Coate, S.: Understanding welfare stigma: taxpayer resentment and statistical discrimination. J. Public Econ. 48(2), 165-183 (1992). https://doi.org/10.1016/0047-2727(92)90025-B

Biewen, M., Steffes, S.: Unemployment persistence: is there evidence for stigma effects? Econ. Lett. 106(3), 188-190 (2010). https://doi.org/10.1016/j. econlet.2009.11.016

Binggeli, S., Krings, F., Sczesny, S.: Perceived competition explains regional differences in the stereotype content of immigrant groups. Soc. Psychol. 45(1), 62-70 (2014). https://doi.org/10.1027/1864-9335/a000160

Bos, A.E., Pryor, J.B., Reeder, G.D., Stutterheim, S.E.: Stigma: advances in theory and research. Basic App. Soc. Psychol. 35(1), 1-9 (2013). https://doi. org/10.1080/01973533.2012.746147

Brand, J.E.: The far-reaching impact of job loss and unemployment. Annu. Rev. Sociol. 41, 359-375 (2015)

Bretschneider, P.: Stigma and social identity of people who are not in paid employment. Doctoral thesis submitted to the University of Exeter. https ://ore.exeter.ac.uk/repository/handle/10871/17309 (2014). Accessed 20 Mar 2019

Cameron, A.C., Trivedi, P.K.: Microeconometrics using stata. Stata Press, College Station (2010)

Deacon, H.: Towards a sustainable theory of health-related stigma: lessons from the HIV/AIDS literature. J. Community Appl. Soc. Psychol. 16 418-425 (2006)

Eriksson, S., Rooth, D.O.: Do employers use unemployment as a sorting criterion when hiring? Evidence from a field experiment. Am. Econ. Rev. 104(3), 1014-1039 (2014). https://doi.org/10.1257/aer.104.3.1014

Farber, H., Silverman, D., von Wachter, T.: Factors determining callbacks to job applications by the unemployed: an audit study. NBER Working Paper, no. 21689. https://doi.org/10.3386/w21689 (2015)

Feather, N.T.: Expectancy-value approaches: present status and future directions. In: Feather, N.T. (ed.) Expectations and actions: expectancy-value models in psychology. Erlbaum, Hillsdale (1982)

Gangl, M.: Welfare states and the scar effects of unemployment: a comparative analysis of the United States and West Germany. Am. J. Soc. 109(6), 1319-1364 (2004). https://doi.org/10.1086/381902

Ghayad, R.: The jobless trap. Working paper. (2014). http://citeseerx.ist.psu.edu/ viewdoc/summary?doi=10.1.1.692.6736. Accessed $12 \operatorname{Jan} 2017$

Gielen, A.C., van Ours, J.C.: Unhappiness and job finding. Economica 81, 544-565 (2014). https://doi.org/10.1111/ecca.12089

Goffman, E.: Stigma. Notes on the management of spoiled identity. PrenticeHall, New York (1963)

Gurr, T., Jungbauer-Gans, M.: Stigma consciousness among the unemployed and prejudices against them: development of two scales for the 7th wave of the panel study "Labour Market and Social Security (PASS)". J. Labour Mark. Res. 46(4), 335-351 (2013). https://doi.org/10.1007/s1265 $1-013-0144-z$
Gurr, T., Jungbauer-Gans, M.: Eine Untersuchung zu Erfahrungen Betroffener mit dem Stigma Arbeitslosigkeit. Soz. Probl. 28(1), 25-50 (2017). https ://doi.org/10.1007/s41059-017-0028-5

Gurr, T., Unger, S., Jungbauer-Gans, M.: Gehen Sanktionen mit einem höheren Stigmabewusstsein bei Arbeitslosen einher? Z. Sozialr. 64(2), 217-248 (2018). https://doi.org/10.1515/zsr-2018-0012

Guyll, M., Madon, S., Prieto, L., Scherr, K.C.: The potential roles of self-fulfilling prophecies, stigma consciousness and stereotype threat in linking Latino/a ethnicity and educational outcomes. J. Soc. Issues 66(1), 113-130 (2010)

Hansen, H.: Unemployment and marital dissolution: a panel data study of Norway. Eur. Sociol. Rev. 21(2), 135-148 (2005). https://doi. org/10.1093/esr/jci009

Hatzenbuehler, M.L., Phelan, J.C., Link, B.G.: Stigma as a fundamental cause of population health inequalities. Am. J. Public Health 103(5), 813-821 (2013). https://doi.org/10.2105/AJPH.2012.301069

Heckman, J., Borjas, G.: Does unemployment cause future unemployment? Definitions, questions and answers from a continuous time model of heterogeneity and state dependence. Economica 47(187), 247-283 (1980). https://doi.org/10.2307/2553150

Herek, G.M.: Sexual orientation differences as deficits. Science and stigma in the history of American psychology. Perspect. Psychol. Sci. 5(6), 693-699 (2010). https://doi.org/10.1177/1745691610388770

Heslin, P.A., Bell, M.P., Fletcher, P.O.: The devil without and within: a conceptual model of social cognitive processes whereby discrimination leads stigmatized minorities to become discouraged workers. J. Organ. Behav. 33(6), 840-862 (2012). https://doi.org/10.1002/job.1795

Hirseland, A., Ramos Lobato, P.: "Die wollen ja ein bestimmtes Bild vermitteln." Zur Neupositionierung von Hilfeempfängern im aktivierenden Sozialstaat. SWS-Rundschau 54(2), 181-200 (2014)

Hohmeyer, K., Wolff, J.: Of carrots and sticks: the effect of workfare announcements on the job search behaviour and reservation wage of welfare recipients. J. Labour Mark. Res. 52(1), 23 S (2018). https://doi. org/10.1186/s12651-018-0245-9

Jones, L:: Unemployment and social integration: a review. J Sociol. Soc. Welf. 15(4), 161-176 (1988)

Karren, R., Sherman, K.: Layoffs and unemployment discrimination: a new stigma. J. Manag. Psychol. 27(8), 848-863 (2012)

Kerbo, H.R.: The stigma of welfare and a passive poor. Soc. Sci. Res. 60(2), 173-187 (1976). https://doi.org/10.1007/BF02404490

Knabe, A., Fischer, H., Klärner, A.: "Armut" als relationales Konstrukt: Die (Re-)Produktion sozialer Ungleichheiten durch Stigmatisierung und "Kontrollversuche" in sozialen Netzwerken. In: Behrmann, L., Eckert, F., Gefken, A. (eds.) "Doing inequality"-Prozesse sozialer Ungleichheit im Blick qualitativer Sozialforschung, pp. 167-190. Springer, Wiesbaden (2018)

Kroft, K., Lange, F., Notowidigdo, M.: Duration dependence and labor market conditions: evidence from a field experiment. Quat. J. Econ. 128(3), 1123-1167 (2013). https://doi.org/10.1093/qje/qjt015

Krug, G., Eberl, A.: What explains the negative effect of unemployment on health? An analysis accounting for reverse causality. Res. Soc. Stratif. Mobil. 55, 25-39 (2018). https://doi.org/10.1016/j.rssm.2018.03.001

Lang, S., Gross, C.: Einflussfaktoren auf das Stigma-Bewusstsein Arbeitsloser. Unpublished manuscript, Hannover (2017)

LeBel, T.P.: Perceptions of and responses to stigma. Sociol. Compass $\mathbf{2}(2)$, 409-432 (2008). https://doi.org/10.1111/j.1751-9020.2007.00081.x

Leszczensky, L., Wolbring, T.: How to deal with reverse causality using panel data? Recommendations for researchers based on a simulation study. (2018). https://doi.org/10.31235/osf.io/8xb4z

Letkemann, P.: Unemployed professionals, stigma management and derivative stigmata. Work Employ. Soc. 16(3), 511-522 (2002). https://doi. org/10.1111/0022-4537.00202

Linden, P., Reibling, N., Krayter, S.: Lieber krank und arbeitslos als „nur"arbeitslos? Die Auswirkungen der Medikalisierung von arbeitslosen Personen auf Stigmatisierungsprozesse. Z Sozialr. 64(4), 431-461 (2018). https://doi.org/10.1515/zsr-2018-0022

Link, B.G., Phelan, J.C.: Conceptualizing stigma. Annu. Rev. Sociol. 27, 363-385 (2001). https://doi.org/10.1146/annurev.soc.27.1.363

Loewenberg, E.:The destigmatization of public dependency. Soc. Serv. Rev. 55(3), 434-452 (1981). https://doi.org/10.1086/643943 
Markowitz, F.E.: The effects of stigma on the psychological well-being and life satisfaction of persons with mental illness. J. Health Soc. Behav. $\mathbf{3 9}$ 335-348 (1998). https://doi.org/10.2307/2676342

Mattocks, K.M., Sullivan, J.C., Bertrand, C., Kinney, R.L., Sherman, M.D., Gustason, C.: Perceived stigma, discrimination, and disclosure of sexual orientation among a sample of lesbian veterans receiving care in the Department of Veterans Affairs. LGBT Health 2(2), 147-153 (2015). https://doi. org/10.1089/lgbt.2014.0131

McFadyen, R.G.: Attitudes toward the unemployed. Hum. Relat. 51(2), 179-199 (1998). https://doi.org/10.1023/A:1016914319477

Miller, C.T., Kaiser, C.R.: A theoretical perspective on coping with stigma. J. Soc. Issues 57(1), 73-92 (2001). https://doi.org/10.1111/0022-4537.00202

Moffitt, R.: An economic model of welfare stigma. Am. Econ. Rev. 73(5), 1023-1035 (1983)

Mortensen, D.T.: Job search and labor market analysis. In: Ashenfelter, O., Layard, R. (eds.) Handbook of labor economics, pp. 849-919. Elsevier, Amsterdam (1986)

Mosley, T.M., Rosenberg, J.: Stigma consciousness and perceived stereotype threat and their effects on academic performance. Univ. Alabama McNair J. 7, 85-114 (2007)

Mousteri, V., Daly, M., Delaney, L.: The scarring effect of unemployment on psychological well-being across Europe. Soc. Sci. Res. 72, 146-169 (2018). https://doi.org/10.1016/j.ssresearch.2018.01.007

Nickell, S.: Biases in dynamic models with fixed effects. Econometrica 49(6), 1417-1426 (1981). https://doi.org/10.2307/1911408

Nunley, J.M., Pugh, A., Romero, N., Seals, R.A.: The effects of unemployment and underemployment on employment opportunities: results from a correspondence audit of the labor market for college graduates. ILR Rev. 70(3), 642-669 (2017). https://doi.org/10.1177/0019793916654686

Nüß, P.: Duration dependence as an unemployment stigma: Evidence from a field experiment in Germany. IMK Working Paper, no. 184 (2017)

Oberholzer-Gee, F.: Nonemployment stigma as rational herding: a field experiment. J. Econ. Behav. Organ. 65, 30-40 (2008). https://doi.org/10.1016/j. jebo.2004.05.008

O'Donnell, A.T., Corrigan, F., Gallagher, S.: The impact of anticipated stigma on psychological and physical health problems in the unemployed group. Front. Psychol. 6, 1263 (2015). https://doi.org/10.3389/fpsyg .2015 .01263

Omori, Y.: Stigma effects on nonemployment. Econ. Inq. 35, 394-416 (1997). https://doi.org/10.1111/j.1465-7295.1997.tb01918.x

Oschmiansky, F., Schmid, G., Kull, S.: Faule Arbeitslose? Leviathan 31(1), 3-31 (2003). https://doi.org/10.1007/s11578-003-0001-5

Paugam, S., Russell, H.: The effects of employment precarity and unemployment on social isolation. In: Duncan, G., Serge, P. (eds.) Welfare regimes and the experience of unemployment in Europe, pp. 243-264. Oxford University Press, Oxford (2000)

Pinel, E.C.: Stigma consciousness: the psychological legacy of social stereotypes. J. Pers. Soc. Psychol. 76(1), 114-128 (1999). https://doi. org/10.1037//0022-3514.76.1.114

Pinel, E.C., Paulin, N.: Stigma consciousness at work. Basic Appl. Soc. Psychol. 27(4), 345-352 (2005). https://doi.org/10.1207/s15324834basp2704_7

Pinel, E.C., Warner, L.R., Chua, P.-P.: Getting there is only half the battle: stigma consciousness and maintaining diversity in higher education. J. Soc. Issues 61(3), 481-506 (2005)

Pryor, J.B., Reeder, G.D.: HIV-related stigma. In: Hall, J.C., Hall, B.J., Cockerell, C.J. (eds.) HIV/AIDS in the post-HAART era: manifestations, treatment, and epidemiology, pp. 790-806. People's Medical Publishing House USA, Raleigh (2011)

Rammstedt, B., John, O.P.: Short version of the Big Five Inventory (BFI-K): development and validation of an economic inventory for assessment of the five factors of personality. Diagnostica 51, 195-206 (2005). https://doi. org/10.1026/0012-1924.51.4.195

Rantakeisu, U., Starrin, B., Hagquist, C.: Financial hardship and shame: a tentative model to understand the social and health effects of unemployment. Br. J. Soc. Work 29(6), 877-901 (1999). https://doi.org/10.1093/ bjsw/29.6.877

Rebien, M., Rothe, T.H.: Langzeitarbeitslose Bewerber aus betrieblicher Perspektive: Zuverlässigkeit ist wichtiger als fachliche Qualifikation. IABKurzbericht. 12 (2018)
Rosenfield, S.: Labeling and mental illness: the effects of received services and perceived stigma on life satisfaction. Am. Sociol. Rev. 62(4), 660-672 (1997). https://doi.org/10.2307/2657432

Rubin, D.B.: Multiple imputation for nonresponse in surveys. Wiley, New York (1987)

Ryan, R.M., Deci, E.L.: Self-determination theory and the facilitation of intrinsic motivation, social development, and well-being. Am. Psychol. 55(1), 68-78 (2000)

Scambler, G.: Health-related stigma. Sociol. Health IIIn. 31(3), 441-455 (2009). https://doi.org/10.1111/j.1467-9566.2009.01161.x

Schels, B., Bethmann, A.: Job search of men and women on long-term social welfare. Int. J. Sociol. Soc. Policy 38(3/4), 224-241 (2018). https://doi. org/10.1108/IJSSP-07-2017-0090

Schwarzer, R., Jerusalem, M.: Generalized self-efficacy scale. In: Weinman, J., Wright, S., Johnston, M. (eds.) Measures in health psychology: a user's portfolio. Causal and control beliefs, pp. 35-37. NFER-Nelson, Windsor (1995)

Schwarzer, R., Jerusalem, M. (eds.). Skalen zur Erfassung von Lehrer- und Schülermerkmalen. Dokumentation der psychometrischen Verfahren im Rahmen der Wissenschaftlichen Begleitung des Modellversuchs Selbstwirksame Schulen. Berlin: Freie Universität Berlin. http://userpage. fu-berlin.de/gesund/skalen/Allgemeine_Selbstwirksamkeit/allgemeine _selbstwirksamkeit.htm (1999). Accessed 25 Mar 2019

Seligman, M.E.: Helplessness: on depression, development, and death. A series of books in psychology. WH Freeman/Times Books/Henry Holt \& Co., New York (1975)

Sherman, J.: Surviving the great recession: growing need and the stigmatized safety net. Soc. Probl. 60, 409-432 (2013). https://doi.org/10.1525/ sp.2013.60.4.409

Sigelman, L., Tuch, S.A.: Metastereotypes. Blacks' perceptions of whites' stereotypes of blacks. Public Opin. Q. 61(1), 87-101 (1997)

Stuber, J., Schlesinger, M.: Sources of stigma for means-tested government programs. Soc. Sci. Med. 63(4), 933-945 (2006). https://doi.org/10.1016/j. socscimed.2006.01.012

Tajfel, H., Turner, J.: An integrative theory of intergroup conflict. In: Austin, W.G., Worchel, S. (eds.) The social psychology of intergroup relations, pp. 33-48. Brooks/Cole, Monterey (1979)

Taylor, D.M., Wright, S.C., Porter, L.E.: Dimensions of perceived discrimination: the personal/group discrimination discrepancy. In: Zanna, M.P., Olson, J.M. (eds.) The psychology of prejudice: the Ontario symposium, vol. 7, pp. 233-255. Psychology Press, London (1994)

Trappmann, M., Beste, J., Bethmann, A., Müller, G.: The PASS panel survey after six waves. J. Labour Mark. Res. 46(4), 275-281 (2013). https://doi. org/10.1007/s12651-013-0150-1

Vaisey, S., Miles, A.: What you can—and can't—do with three-wave panel data. Sociol. Method Res. 46(1), 44-67 (2017). https://doi.org/10.1177/00491 24114547769

van Belle, E., Caers, R., De Couck, M., DiStasio, V., Baert S.: Why is unemployment duration a sorting criterion in hiring? IZA Discussion Paper, vol. 10876. IZA, Bonn (2017)

van den Berg, G., van Ours, J.: Unemployment dynamics and duration dependence. J. Labor Econ. 14(1), 100-125 (1996). https://doi.org/10.1086/20980 5

Vishwanath, T.: Job search, stigma effect, and escape Rate from unemployment. J. Labor Econ. 7(4), 487-502 (1989). https://doi.org/10.1086/298218

Vroom, V.H.: Work and motivation. Wiley, New York (1964)

Wang, K., Stroebe, K., Dovidio, J.F.: Stigma consciousness and prejudice ambiguity: can it be adaptive to perceive the world as biased? Personal. Individ. Differ. 53, 241-245 (2012). https://doi.org/10.1016/j.paid.2012.03.021

Yaniv, G.: Welfare fraud and welfare stigma. J. Econ. Psychol. 18(4), 435-451 (1997). https://doi.org/10.1016/S0167-4870(97)00016-0

\section{Publisher's Note}

Springer Nature remains neutral with regard to jurisdictional claims in published maps and institutional affiliations. 